Research Article

\title{
The Existence of Zero Waves for Nonsimplified Chromatography System
}

\author{
Maozhou Lin (D), Lihui Guo (D), and Yinsong Bai \\ College of Mathematics and System Sciences, Xinjiang University, Urumqi 830046, China \\ Correspondence should be addressed to Lihui Guo; lihguo@126.com
}

Received 11 November 2019; Revised 2 January 2020; Accepted 6 January 2020; Published 12 February 2020

Academic Editor: Aimé Lay-Ekuakille

Copyright (C) 2020 Maozhou Lin et al. This is an open access article distributed under the Creative Commons Attribution License, which permits unrestricted use, distribution, and reproduction in any medium, provided the original work is properly cited.

In this paper, we mainly consider Riemann problem for the widely used nonsimplified chromatography system with initial data consisting of three pieces of constant states. Through phase plane analysis, the solutions of the nonsimplified chromatography system are established. When the different initial data tend to -1 from the right side, the existence of zero shock wave, zero delta shock wave, and zero rarefaction wave is obtained via analyzing its wave interaction. Finally, the correctness of the main conclusions is verified by numerical simulation, and the numerical results are in good agreement with the theoretical solutions of several experimental cases.

\section{Introduction}

Chromatography plays an important role in modern industry. It is used to separate two chemical components especially by chemists and engineers. The research of the nonlinear chromatography system is, in particular, a significant part of the theory of chromatography. Mazzotti [1-3] captured the delta shock wave for local equilibrium model (1) by numerical and experimental methods:

$$
\left\{\begin{array}{l}
u_{t}+\left(u+\frac{a u}{1-u+v}\right)_{x}=0, \\
v_{t}+\left(v+\frac{b v}{1-u+v}\right)_{x}=0,
\end{array}\right.
$$

where $b>a>0$ are constants. $u$ and $v$, which describe concentrations of two chemical species, are positive function with $1-u+v>0$. The complexity of system (1) is due to hyperbolicity in the region $a(1+v)+b(1-u)^{2}-$ $4 a b(1-u+v)>0$ and ellipticity in the rest of the phase plane.

In order to make the deep research for (1), one could consider the following chromatography system:

$$
\left\{\begin{array}{l}
u_{t}+\left(\frac{u}{1-u+v}\right)_{x}=0 \\
v_{t}+\left(\frac{v}{1-u+v}\right)_{x}=0 .
\end{array}\right.
$$

In 2013, Wang [4] had found the Riemann solution of (2) contains delta shock wave with Dirac delta function support on both $u$ and $v$. It is clear that (2) belongs to the Temple class $[5,6]$; in other words, the shock curves and rarefaction curves are connected in the phase plane. One can refer to [7-13].

Recently, Ambrosio et al. [14] introduced the change of variables:

$$
\begin{aligned}
& p=v-u, \\
& q=v+u,
\end{aligned}
$$

and derived the following simplified chromatography system:

$$
\left\{\begin{array}{l}
p_{t}+\left(\frac{p}{1+p}\right)_{x}=0 \\
q_{t}+\left(\frac{q}{1+p}\right)_{x}=0
\end{array}\right.
$$


They obtain the well-posedness. In 2011, Sun [15] discovered that solutions of (4) and (6) contain delta shock wave in case of $p_{-}=0<p_{+}$via self-similar vanishing viscosity approach. In 2015, Shen [16] considered the asymptotic behaviors of solutions of the perturbed Riemann problem (4) and (6) near the singular curve. For other related results, one can refer to [17-24].

The zero waves describe the equilibrium of separation; under this state, there is no exchange of kinetic between two chemical species. Zero waves play an important role in analyzing the chromatography systems [1, 2, 23]. Moreover, it plays a key role in the study of traffic flow, production flow, and the supply chain with limited buffers [25-29].

At the same time, solving partial differential equations is always an interesting and difficult task. In recent decades, many kinds of analytical methods have been proposed. There are also some numerical methods, which are powerful treatments in solving nonlinear equations. Such as numerical methods of two-phase flow equations are proposed and developed by Zeidan et al. [30-34], which provide perfect approaches for the study of wave structure and our future studies. In this paper, we construct a solution of (2) with Riemann initial data:

$$
(u, v)(x, t)=\left\{\begin{array}{lc}
\left(u_{-}, v_{-}\right), & -\infty<x<0, \\
\left(u_{m}, v_{m}\right), & 0<x<1, \\
\left(u_{+}, v_{+}\right), & 1<x<+\infty
\end{array}\right.
$$

and analyse the interaction of various waves. We find interesting phenomena: when the differences $v_{-}-u_{-}$and $v_{m}-$ $u_{m}$ in $(5)$ tend to $(-1)^{+}$, the zero waves occur. Now, we give our main results.

Theorem 1. According to different zero waves produced, we divide the solution of (2) and (5) into three types:

(1) When $-1<v_{-}-u_{-}<v_{m}-u_{m}<0$, on the one hand, the solution performs as a zero shock wave if $v_{+}-$ $u_{+}<0$ and $\left(v_{-}-u_{-}\right) \longrightarrow(-1)^{+}$. On the other hand, its solution performs as a zero shock wave in the interval $[0,1]$ and a zero delta shock wave in the interval $(1, \infty)$ if $v_{+}-u_{+}>0$ and $\left(v_{-}-u_{-}\right) \longrightarrow$ $(-1)^{+}$

(2) When $-1<v_{-}-u_{-}<0<v_{m}-u_{m}$ and $v_{-}-u_{-}<$ $v_{+}-u_{+}$, the solution performs as a zero delta shock wave when $\left(v_{-}-u_{-}\right) \longrightarrow(-1)^{+}$.

(3) When $-1<v_{m}-u_{m}<0$ and $v_{m}-u_{m}<\min \left\{v_{-}-u_{-}\right.$, $\left.v_{+}-u_{+}\right\}$, a zero rarefaction wave is alive in the interval $[0,1]$ when $\left(v_{m}-u_{m}\right) \longrightarrow(-1)^{+}$.

This paper is divided into seven sections. In Section 2, we review the Riemann problem (2) and (6) and discover the zero waves appear when the differences $v_{-}-u_{-}$and $v_{+}-u_{+}$ in $(5)$ tend to $(-1)^{+}$. In Section 3 , we construct solution of Riemann problem (2) and (5), and then we obtain zero shock wave by studying the interaction of waves. In Section 4 and Section 5, we obtain, by analogy, zero delta shock wave and zero rarefaction wave of system (2) and (5). In Section 6, we verify the presentation of zero waves by numerical simulations. Finally, conclusions are drawn in Section 7.

\section{Zero Waves in the Limit of Riemann Solutions to (2)}

In this section, we review the solution to system (2) with initial data:

$$
(u, v)(x, 0)= \begin{cases}\left(u_{-}, v_{-}\right), & x<0, \\ \left(u_{+}, v_{+}\right), & x>0\end{cases}
$$

where $u_{ \pm}$and $v_{ \pm}$are positive constants. In light of [4], we know characteristics satisfy $\lambda_{1}=1 /(1-u+v)^{2}$ and $\lambda_{2}=1 /(1-u+v)$. System (2) is strictly hyperbolic if $-1<v-u<0$ or $v-u>0$ and nonstrictly hyperbolic if $v-u=0$. The characteristics $\lambda_{1}$ and $\lambda_{2}$ are genuinely nonlinear and linearly degenerate, respectively. Therefore, the associated waves are rarefaction waves $R$ or shock waves $S$ for $\lambda_{1}$ and contact discontinuities $J$ for $\lambda_{2}$ :

$$
\begin{aligned}
& R:\left\{\begin{array}{l}
\frac{u}{u_{-}}=\frac{v}{v_{-}}, \quad v+u<v_{-}-u_{-}, \\
\lambda=\frac{1}{(1-u+v)^{2}},
\end{array}\right. \\
& S:\left\{\begin{array}{l}
\frac{u}{u_{-}}=\frac{v}{v_{-}}, \quad v+u>v_{-}-u_{-}, \\
\omega=\frac{1}{(1-u+v)\left(1-u_{-}+v_{-}\right)}
\end{array}\right. \\
& J:\left\{\begin{array}{l}
v-u=v_{-}-u_{-}, \\
\tau=\frac{1}{1-u+v}=\frac{1}{1-u_{-}+v_{-}} .
\end{array}\right.
\end{aligned}
$$

Using the phase plane analysis method (see Figure 1), Riemann solutions of (2) are constructed as follows:

(1) If $0<v_{-}-u_{-}<v_{+}-u_{+}$, then the Riemann solution is $\overleftarrow{S}+J$

$$
(u, v)(x, t)= \begin{cases}\left(u_{-}, v_{-}\right), & \xi<\omega, \\ \left(\frac{\left(v_{+}-u_{+}\right) u_{-}}{v_{-}-u_{-}}, \frac{\left(v_{+}-u_{+}\right) v_{-}}{v_{-}-u_{-}}\right), & \omega<\xi<\tau, \\ \left(u_{+}, v_{+}\right), & \xi>\tau,\end{cases}
$$

in which the propagation speeds of $\overleftarrow{S}$ and $J$ are $\omega=$ $1 /\left(\left(1-u_{-}+v_{-}\right)\left(1-u_{+}+v_{+}\right)\right)$and $\tau=1 /\left(1-u_{+}+v_{+}\right)$, respectively. In particular, if $v_{+}-u_{+}=0$, then $\omega=\tau=1$, and the line of $\overleftarrow{S}$ is identical with $J$ Therefore, there exists the composite wave $\overleftarrow{S} J$.

(2) If $0<v_{+}-u_{+}<v_{-}-u_{-}$, then solution is $\overleftarrow{R}+J$ : 


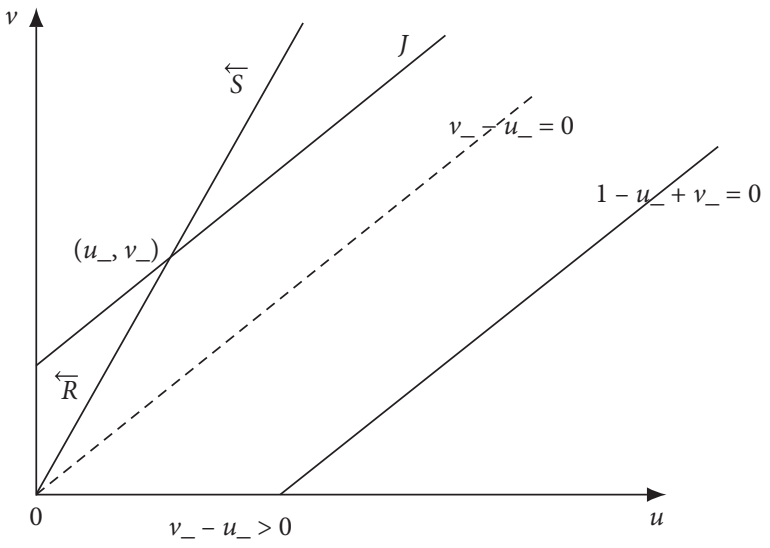

(a)

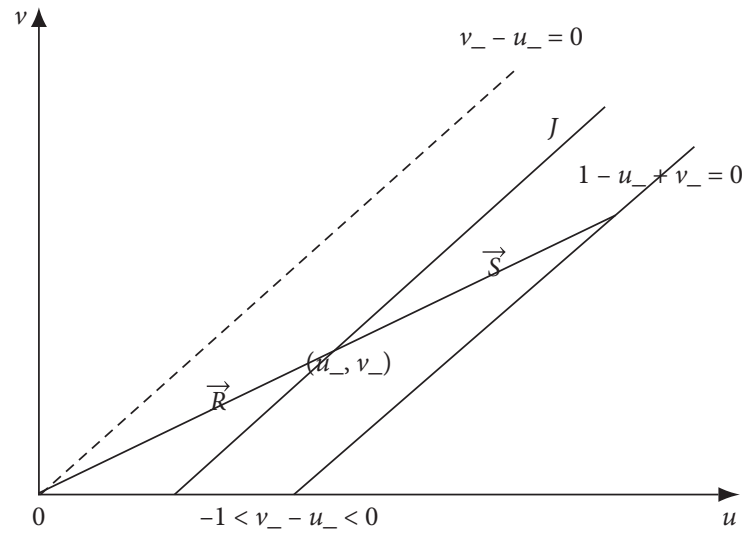

(b)

Figure 1: Phase plane.

$$
(u, v)(x, t)= \begin{cases}\left(u_{-}, v_{-}\right), & \xi<\lambda_{1}\left(u_{-}, v_{-}\right), \\ \frac{u}{u_{-}}=\frac{v}{v_{-}}, & \lambda_{1}\left(u_{-}, v_{-}\right)<\xi<\lambda_{1}\left(u_{+}, v_{+}\right), \\ \left(\frac{\left(v_{+}-u_{+}\right) u_{-}}{v_{-}-u_{-}}, \frac{\left(v_{+}-u_{+}\right) v_{-}}{v_{-}-u_{-}}\right), & \lambda_{1}\left(u_{-}, v_{-}\right)<\xi<\tau, \\ \left(u_{+}, v_{+}\right), & \xi>\tau .\end{cases}
$$

If $v_{+}-u_{+}=0$, then a composite wave $\overleftarrow{R} J$ forms

(3) If $-1<v_{-}-u_{-}<v_{+}-u_{+}<0$, then the Riemann solution is $J+\vec{S}$ :

$$
(u, v)(x, t)= \begin{cases}\left(u_{-}, v_{-}\right), & \xi<\tau, \\ \left(\frac{\left(v_{-}-u_{-}\right) u_{+}}{v_{+}-u_{+}}, \frac{\left(v_{-}-u_{-}\right) v_{+}}{v_{+}-u_{+}}\right), & \tau<\xi<\omega, \\ \left(u_{+}, v_{+}\right), & \xi>\omega .\end{cases}
$$

in which the propagation speeds of $J$ and $S$ are $\tau=$ $1 /\left(1-u_{-}+v_{-}\right)$and $\omega=1 /\left(\left(1-u_{-}+v_{-}\right)\left(1-u_{+}+v_{+}\right)\right)$, respectively.

$$
\begin{aligned}
\lim _{\left(v_{-}-u_{-}\right) \longrightarrow(-1)^{+}} \tau= & \lim _{\left(v_{-}-u_{-}\right) \longrightarrow(-1)^{+}} \frac{1}{1-u_{-}+v_{-}}=+\infty \\
\lim _{\left(v_{-}-u_{-}\right) \longrightarrow(-1)^{+}} \omega & =\lim _{\left(v_{-}-u_{-}\right) \longrightarrow(-1)^{+}} \frac{1}{\left(1-u_{-}+v_{-}\right)\left(1-u_{+}+v_{+}\right)}=+\infty
\end{aligned}
$$


This fact in turn implies that both the propagation speeds of $J$ and $\vec{S}$ tend to $+\infty$. In other words, both $J$ and $\vec{S}$ coincide with the positive $x$-axis and the middle state $\left(\left(v_{-}-u_{-}\right) u_{+} /\left(v_{+}-u_{+}\right), \quad\left(v_{-}-u_{-}\right) v_{+} /\right.$ $\left.\left(v_{+}-u_{+}\right)\right)$disappears. Thus, zero shock wave [28] appears in this situation.

(4) If $-1<v_{+}-u_{+}<0<v_{-}-u_{-}$, then the solution is $J+\vec{R}$ :

$$
(u, v)(x, t)= \begin{cases}\left(u_{-}, v_{-}\right), & \xi<\tau, \\ \left(\frac{\left(v_{-}-u_{-}\right) u_{+}}{v_{+}-u_{+}}, \frac{\left(v_{-}-u_{-}\right) v_{+}}{v_{+}-u_{+}}\right), & \tau<\xi<\lambda_{1}\left(u_{-}, v_{-}\right), \\ \frac{u}{u_{+}}=\frac{v}{v_{+}}, & \lambda_{1}\left(u_{-}, v_{-}\right)<\xi<\lambda_{1}\left(u_{+}, v_{+}\right), \\ \left(u_{+}, v_{+}\right), & \xi>\lambda_{1}\left(u_{+}, v_{+}\right) .\end{cases}
$$

in which $\tau=1 /\left(1-u_{-}+v_{-}\right)$. Since $\left(v_{+}-u_{+}\right) \longrightarrow$ $(-1)^{+}$, then

$$
\begin{aligned}
\lim _{\left(v_{+}-u_{+}\right) \longrightarrow(-1)^{+}} \lambda_{1}\left(u_{+}, v_{+}\right) & =\lim _{\left(v_{+}-u_{+}\right) \longrightarrow(-1)^{+}} \frac{1}{\left(1-u_{+}+v_{+}\right)^{2}} \\
& =+\infty .
\end{aligned}
$$

Equality (13) implies the propagation speed of the wave front of $\vec{R}$ tends to $+\infty$. Then, zero rarefaction wave forms.

(5) If $-1<v_{+}-u_{+}<v_{-}-u_{-}<0$, then the Riemann solution is $\overleftarrow{R}+\vec{R}$

$$
(u, v)(x, t)= \begin{cases}\left(u_{-}, v_{-}\right), & \xi<\lambda_{1}\left(u_{-}, v_{-}\right) \\ \frac{u}{u_{-}}=\frac{v}{v_{-}}, & \lambda_{1}\left(u_{-}, v_{-}\right)<\xi<1, \\ \frac{u}{u_{+}}=\frac{v}{v_{+}}, & 1<\xi<\lambda_{1}\left(u_{+}, v_{+}\right), \\ \left(u_{+}, v_{+}\right), & \xi>\lambda_{1}\left(u_{+}, v_{+}\right) .\end{cases}
$$

The line is $x=t$ both the wave front of $\overleftarrow{R}$ and the wave back of $\vec{R}$. As $\left(v_{+}-u_{+}\right) \longrightarrow(-1)^{+}$, the zero rarefaction wave also appears in this condition.

(6) If $-1<v_{-}-u_{-}<0<v_{+}-u_{+}$, Wang [4] constructed delta shock wave:

$(u, v)(x, t)= \begin{cases}\left(u_{-}, v_{-}\right), & x<\sigma t, \\ (\beta(t), \beta(t)) \delta(x-\sigma t), & x=\sigma t, \\ \left(u_{+}, v_{+}\right), & x>\sigma t,\end{cases}$

where

$$
\begin{aligned}
\sigma & =\frac{1}{\left(1-u_{-}+v_{-}\right)\left(1-u_{+}+v_{+}\right)}, \\
\beta(t) & =\frac{\left(u_{-} v_{+}-u_{+} v_{-}\right) t}{\left(1-u_{-}+v_{-}\right)\left(1-u_{+}+v_{+}\right)} .
\end{aligned}
$$

He checked that the measure solution (15) with (16) satisfies the generalized Rankine-Hugoniot condition:

$$
\left\{\begin{array}{l}
\frac{d x}{d t}=\sigma \\
\frac{d \beta(t)}{d t}=-\sigma[u]+\left[\frac{u}{1-u+v}\right] \\
\frac{d \beta(t)}{d t}=-\sigma[v]+\left[\frac{v}{1-u+v}\right]
\end{array}\right.
$$

To ensure the uniqueness of delta shock wave solution, entropy condition should be satisfied provided $-1<v_{-}-u_{-}<$ $0<v_{+}-u_{+}$,

$$
\lambda_{1}\left(u_{+}, v_{+}\right)<\lambda_{2}\left(u_{+}, v_{+}\right)<\sigma<\lambda_{2}\left(u_{-}, v_{-}\right)<\lambda_{1}\left(u_{-}, v_{-}\right),
$$

namely,

$$
\begin{aligned}
\frac{1}{\left(1-u_{+}+v_{+}\right)^{2}} & <\frac{1}{1-u_{+}+v_{+}}<\sigma<\frac{1}{1-u_{-}+v_{-}} \\
& <\frac{1}{\left(1-u_{-}+v_{-}\right)^{2}} .
\end{aligned}
$$

That is to say, all the characteristics are incoming from both sides.

For the measure solution (15) with (16), we deduce 


$$
\begin{aligned}
\lim _{\left(v_{-}-u_{-}\right) \longrightarrow(-1)^{+}} \sigma & =\lim _{\left(v_{-}-u_{-}\right) \rightarrow(-1)^{+}} \frac{1}{\left(1-u_{-}+v_{-}\right)\left(1-u_{+}+v_{+}\right)} \\
& =+\infty, \\
\lim _{\left(v_{-} u_{-}\right) \rightarrow(-1)^{+}} \beta(t) & =\lim _{\left(v_{-}-u_{-}\right) \rightarrow(-1)^{+}} \frac{\left(u_{-} v_{+}-u_{+} v_{-}\right) t}{\left(1-u_{-}+v_{-}\right)\left(1-u_{+}+v_{+}\right)} \\
& =+\infty .
\end{aligned}
$$

We apply (20) and (21) to deduce that both the propagation speed and the strength of the delta shock wave tend to $+\infty$. Therefore, there is a delta shock wave.

\section{Analysis of Zero Shock Wave}

Based on the fact that all situations occurring in dynamic pictures cannot be depicted by Riemann problem (2), we consider the initial valuing problem (2) and (5).

In this section, we are interested in the interactions of zero shock wave with other elementary waves. Initial data with (5) together with $-1<v_{-}-u_{-}<-1+\varepsilon<v_{m}-u_{m}<0$ ensure the appearance of zero shock wave at the point $(0,0)$.

Case 1. $-1<v_{-}-u_{-}<v_{+}-u_{+}<v_{m}-u_{m}<0$.

For small $t>0$, if $-1<v_{-}-u_{-}<v_{+}-u_{+}<v_{m}-u_{m}<0$ (Figure 2), then the solution of (2) and (5) is as follows:

$$
\begin{aligned}
& \left(u_{-}, v_{-}\right)+J_{1}+\left(u_{1}, v_{1}\right)+\overrightarrow{S_{1}}+\left(u_{m}, v_{m}\right)+J_{2}+\left(u_{2}, v_{2}\right) \\
& \quad+\vec{R}+\left(u_{+}, v_{+}\right),
\end{aligned}
$$

in which

$$
\begin{aligned}
& \left(u_{1}, v_{1}\right)=\left(\frac{\left(v_{-}-u_{-}\right) u_{m}}{v_{m}-u_{m}}, \frac{\left(v_{-}-u_{-}\right) v_{m}}{v_{m}-u_{m}}\right), \\
& \left(u_{2}, v_{2}\right)=\left(\frac{\left(v_{m}-u_{m}\right) u_{+}}{v_{+}-u_{+}}, \frac{\left(v_{m}-u_{m}\right) v_{+}}{v_{+}-u_{+}}\right) .
\end{aligned}
$$

The propagation speeds of $\overrightarrow{S_{1}}$ and $J_{2}$ are $\omega_{1}=1 /((1-$ $\left.\left.u_{-}+v_{-}\right)\left(1-u_{m}+v_{m}\right)\right)$ and $\tau_{2}=1 /\left(1-u_{m}+v_{m}\right)$, respectively. Since $\omega_{1}>\tau_{2}$ provided $-1<u_{-}-v_{-}<0$, then the shock wave $\overrightarrow{S_{1}}$ overtakes contact discontinuity $J_{2}$ in finite time. The waves $\overrightarrow{S_{1}}$ and $J_{2}$ interact at the point:

$$
\left(x_{1}, t_{1}\right)=\left(\frac{1}{u_{-}-v_{-}}, \frac{\left(1-u_{m}+v_{m}\right)\left(1-u_{-}+v_{-}\right)}{u_{-}-v_{-}}\right) .
$$

For $t>t_{1}$, notice the condition $-1<v_{1}-u_{1}<v_{2}-u_{2}<0$, a new contact discontinuity $J_{3}$ and a new shock $\overrightarrow{S_{2}}$ generate. By applying (23), the following equation can be obtained:

$$
\left(u_{3}, v_{3}\right)=\left(\frac{\left(v_{-}-u_{-}\right) u_{+}}{v_{+}-u_{+}}, \frac{\left(v_{-}-u_{-}\right) v_{+}}{v_{+}-u_{+}}\right) .
$$

The propagation speeds of $\overrightarrow{S_{2}}$ and the wave back in $\vec{R}$, respectively, are $\omega_{2}=1 /\left(\left(1-u_{-}+v_{-}\right)\left(1-u_{m}+v_{m}\right)\right)$ and

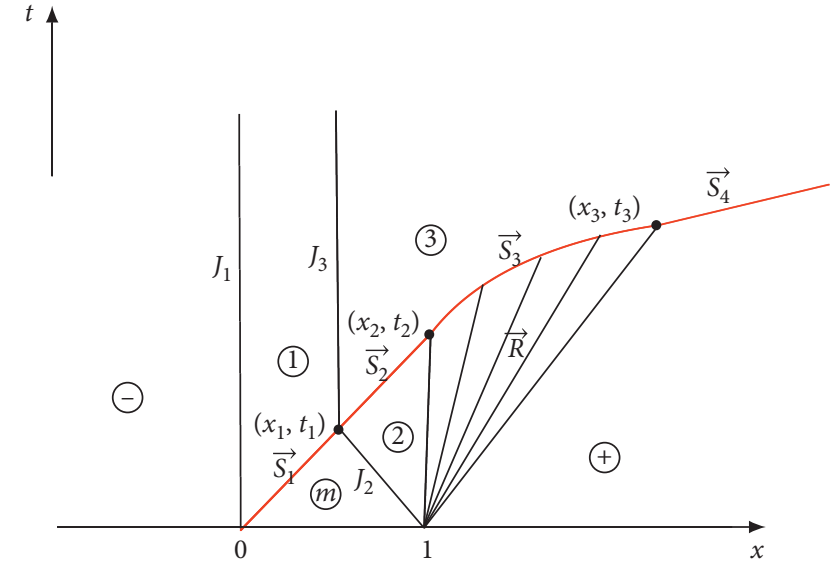

Figure 2: When $-1<v_{-}-u_{-}<v_{+}-u_{+}<v_{m}-u_{m}<0$.

$\lambda_{2}\left(u_{2}, v_{2}\right)=1 /\left(1-u_{m}+v_{m}\right)^{2}$. Since $\omega_{2}>\lambda_{2}\left(u_{2}, v_{2}\right), \overrightarrow{S_{2}}$ can catch up with $\vec{R}$ in finite time and the intersection point $\left(x_{2}, t_{2}\right)$ is given by

$$
\left(x_{2}, t_{2}\right)=\left(\frac{1-u_{m}+v_{m}}{v_{m}-u_{m}-\left(v_{-}-u_{-}\right)}, \frac{\left(1-u_{-}+v_{-}\right)\left(1-u_{m}+v_{m}\right)^{2}}{v_{m}-u_{m}-\left(v_{-}-u_{-}\right)}\right) .
$$

After $t_{2}$, the shock wave $\overrightarrow{S_{3}}$ crosses the rarefaction wave $\vec{R}$ with a varying speed during the penetration. The shock wave $\overrightarrow{S_{3}}$ satisfies the following equation:

$$
\left\{\begin{array}{l}
\frac{d x}{d t}=\frac{1}{\left(1-u_{-}+v_{-}\right)(1-u+v)}, \\
x-1=\frac{t}{(1-u+v)^{2}}, \\
\left(x_{2}, t_{2}\right)=\left(\frac{1-u_{m}+v_{m}}{v_{m}-u_{m}-\left(v_{-}-u_{-}\right)}, \frac{\left(1-u_{-}+v_{-}\right)\left(1-u_{m}+v_{m}\right)^{2}}{v_{m}-u_{m}-\left(v_{-}-u_{-}\right)}\right) .
\end{array}\right.
$$

In view of (27), we can obtain that

$$
\sqrt{x-1}=\frac{\sqrt{t}}{1-u_{-}+v_{-}}-\sqrt{\frac{v_{m}-u_{m}-\left(v_{-}-u_{-}\right)}{1-u_{-}+v_{-}}} .
$$

The wave $S_{3}$ and the wave front of $\vec{R}$ interact at the point

$$
\begin{aligned}
\left(x_{3}, t_{3}\right)= & \left(1+\frac{\left(v_{m}-u_{m}-\left(v_{-}-u_{-}\right)\right)\left(1-u_{-}+v_{-}\right)}{\left(v_{+}-u_{+}-\left(v_{-}-u_{-}\right)\right)^{2}},\right. \\
& \left.\frac{\left(v_{m}-u_{m}-\left(v_{-}-u_{-}\right)\right)\left(1-u_{-}+v_{-}\right)\left(1-u_{+}+v_{+}\right)^{2}}{\left(v_{+}-u_{+}-\left(v_{-}-u_{-}\right)\right)^{2}}\right) .
\end{aligned}
$$

After the time $t_{3}$, a new shock wave $\overrightarrow{S_{4}}$ forms and the propagation speed of $\overrightarrow{S_{4}}$ is $1 /\left(1-u_{+}+v_{+}\right)\left(1-u_{-}+v_{-}\right)$.

Combining (24), (26), and (29), we discover 


$$
\begin{aligned}
\lim _{\left(v_{-}-u_{-}\right) \longrightarrow(-1)^{+}}\left(x_{1}, t_{1}\right) & =\lim _{\left(v_{-}-u_{-}\right) \longrightarrow(-1)^{+}}\left(x_{2}, t_{2}\right) \\
& =\lim _{\left(v_{-}-u_{-}\right) \longrightarrow(-1)^{+}}\left(x_{3}, t_{3}\right)=(1,0) .
\end{aligned}
$$

Based on the above discussion, as $\left(v_{-}-u_{-}\right) \longrightarrow(-1)^{+}$, the solution of (2) and (5) has the zero shock wave starting from the point $(0,0)$.

Case 2. $-1<v_{-}-u_{-}<v_{m}-u_{m}<v_{+}-u_{+}<0$.

In this case, for $-1<v_{-}-u_{-}<v_{m}-u_{m}<v_{+}-u_{+}<0$ (see Figure 3), from Case 1, it is clear to see that the interaction point $\left(x_{1}, t_{1}\right)$ of $\overrightarrow{S_{1}}$ and $J_{2}$ is given by (24), and the states $\left(u_{1}, v_{1}\right),\left(u_{2}, v_{2}\right)$, and $\left(u_{3}, v_{3}\right)$ satisfy (23) and (25), respectively.

Since $\omega_{3}=1 /\left(\left(1-u_{-}+v_{-}\right)\left(1-u_{m}+v_{m}\right)\right)>\omega_{2}=1 /((1-$ $\left.\left.u_{m}+v_{m}\right)\left(1-u_{+}+v_{+}\right)\right)$, for $-1<v_{-}-u_{-}<v_{+}-u_{+}<0$, then $\overrightarrow{S_{3}}$ overtakes the $\overrightarrow{S_{2}}$ in finite time and the interaction point is as follows:

$$
\begin{aligned}
\left(x_{2}, t_{2}\right)= & \left(\frac{1-u_{+}+v_{+}}{v_{+}-u_{+}-\left(v_{-}-u_{-}\right)}\right. \\
& \left.\frac{\left(1-u_{-}+v_{-}\right)\left(1-u_{m}+v_{m}\right)\left(1-u_{+}+v_{+}\right)}{v_{+}-u_{+}-\left(v_{-}-u_{-}\right)}\right) .
\end{aligned}
$$

In view of (24) and (31), we see

$$
\lim _{\left(v_{-}-u_{-}\right) \longrightarrow(-1)^{+}}\left(x_{1}, t_{1}\right)=\lim _{\left(v_{-}-u_{-}\right) \longrightarrow(-1)^{+}}\left(x_{2}, t_{2}\right)=(1,0) .
$$

When $\left(v_{-}-u_{-}\right) \longrightarrow(-1)^{+}$, the propagation speeds of all shock waves and contact discontinuities tend to $+\infty$. Thus, there is a zero shock wave starting from $(0,0)$ in the limit $\left(v_{-}-u_{-}\right) \longrightarrow(-1)^{+}$.

Case 3. $-1<v_{-}-u_{-}<v_{m}-u_{m}<0<v_{+}-u_{+}$.

Our plan is to show that a delta shock wave $\delta S_{1}$ emits from the point $(1,0)$ as $-1<v_{-}-u_{-}<v_{m}-u_{m}<0<v_{+}-u_{+}$ (see Figure 4$)$. The state $\left(u_{1}, v_{1}\right)$ is given by (23).

Owing to $\omega_{1}>\sigma_{1}$, then $S_{1}$ overtakes $\delta S_{1}$ at the point

$$
\begin{aligned}
\left(x_{1}, t_{1}\right)= & \left(\frac{1-u_{+}+v_{+}}{v_{+}-u_{+}-\left(v_{-}-u_{-}\right)},\right. \\
& \left.\frac{\left(1-u_{-}+v_{-}\right)\left(1-u_{m}+v_{m}\right)\left(1-u_{+}+v_{+}\right)}{v_{+}-u_{+}-\left(v_{-}-u_{-}\right)}\right) .
\end{aligned}
$$

It is easy to see that a new delta shock $\delta S_{2}$ forms, with $t>t_{1},-1<v_{-}-u_{-}<v_{m}-u_{m}<0<v_{+}-u_{+}$. The propagation

$$
1
$$

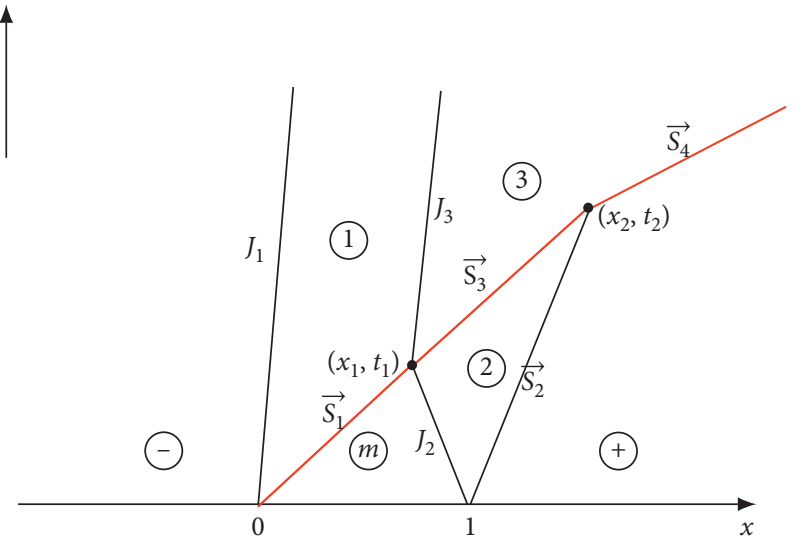

Figure 3: When $-1<v_{-}-u_{-}<v_{m}-u_{m}<v_{+}-u_{+}<0$.

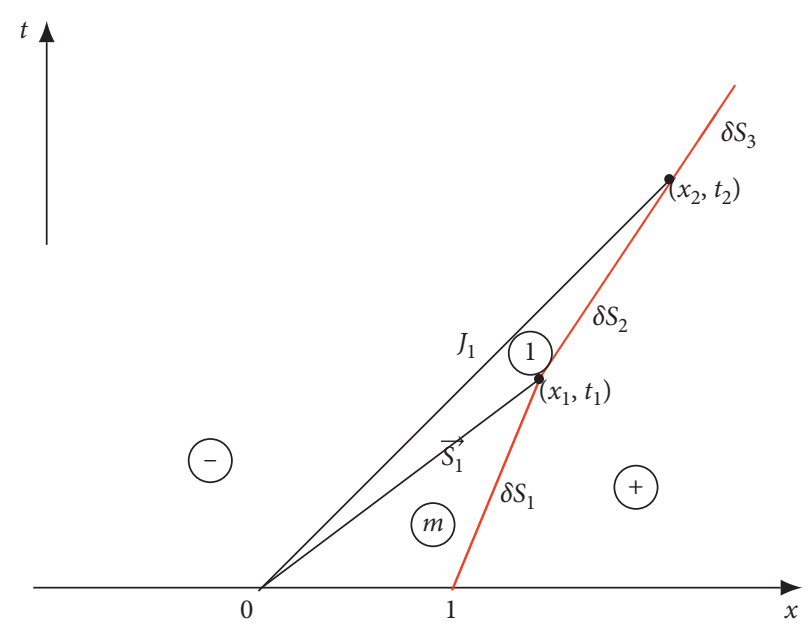

FIGURE 4: When $-1<v_{-}-u_{-}<v_{m}-u_{m}<0<v_{+}-u_{+}$.

speed of $\delta S_{2}$ is $\sigma_{2}=1 /\left(\left(1-u_{-}+v_{-}\right)\left(1-u_{+}+v_{+}\right)\right)$. Notice that propagation speed of contact discontinuity $J$ is $\tau_{1}$ and $\tau_{1}>\sigma_{2}$, and $J_{1}$ and $\delta S_{2}$ interact at the point

$$
\begin{aligned}
\left(x_{2}, t_{2}\right)= & \left(\frac{\left(v_{+}-u_{+}-\left(v_{m}-u_{m}\right)\right)\left(1-u_{+}+v_{+}\right)}{\left(v_{+}-u_{+}-\left(v_{-}-u_{-}\right)\right)\left(v_{+}-u_{+}\right)}\right. \\
& \left.\frac{\left(v_{+}-u_{+}-\left(v_{m}-u_{m}\right)\right)\left(1-u_{-}+v_{-}\right)\left(1-u_{+}+v_{+}\right)}{\left(v_{+}-u_{+}-\left(v_{-}-u_{-}\right)\right)\left(v_{+}-u_{+}\right)}\right) .
\end{aligned}
$$

For $t>t_{2}$, there is a new delta shock $\delta S_{3}$ with same propagation speed of $\delta S_{2}$. It follows from (16) that the strengths of the all delta shock waves satisfy the following equation:

$$
\begin{array}{ll}
\beta_{1}(t)=\frac{u_{m} v_{+}-u_{+} v_{m}}{\left(1-u_{m}+v_{m}\right)\left(1-u_{+}+v_{+}\right)} t, & 0 \leq t \leq t_{1}, \\
\beta_{2}(t)=\beta_{1}\left(t_{1}\right)+\frac{u_{1} v_{+}-u_{+} v_{1}}{\left(1-u_{1}+v_{1}\right)\left(1-u_{+}+v_{+}\right)}\left(t-t_{1}\right), & t_{1} \leq t \leq t_{2}, \\
\beta_{3}(t)=\beta_{2}\left(t_{2}\right)+\frac{u_{-} v_{+}-u_{+} v_{-}}{\left(1-u_{-}+v_{-}\right)\left(1-u_{+}+v_{+}\right)}\left(t-t_{2}\right), & t \geq t_{2} .
\end{array}
$$


We then combine (33) and (34) to discover

$$
\begin{aligned}
\lim _{\left(v_{-}-u_{-}\right) \longrightarrow(-1)^{+}}\left(x_{1}, t_{1}\right) & =\underset{\left(v_{-}-u_{-}\right) \longrightarrow(-1)^{+}}{\lim }\left(x_{2}, t_{2}\right)=(1,0), \\
\lim _{\left(v_{-}-u_{-}\right) \longrightarrow(-1)^{+}} \beta_{1}(t) & =\underset{\left(v_{-}-u_{-}\right) \longrightarrow(-1)^{+}}{\lim } \beta_{2}(t) \\
& =\underset{\left(v_{-}-u_{-}\right) \longrightarrow(-1)^{+}}{\lim } \beta_{3}(t)=\infty .
\end{aligned}
$$

Based on the above analysis, all the waves overlap with the positive $x$-axis when $\left(v_{-}-u_{-}\right) \longrightarrow(-1)^{+}$. Hence, at the interval $[0,1]$ and the point $(1,0)$, zero shock wave and zero delta shock wave occur, respectively.

The above discussions verify the type (1) of Theorem 1.

\section{Analysis of Zero Delta Shock Wave}

In this section, we mainly consider zero delta shock wave interacts with other waves. Taking sufficiently $\varepsilon$ together with $-1<v_{-}-u_{-}<-1+\varepsilon<0<v_{m}-u_{m}$ and $v_{-}-u_{-} \longrightarrow(-1)^{+}$, the Riemann solution of (2) has a zero delta shock wave at the point $(0,0)$.

Case 4. $-1<v_{-}-u_{-}<0<v_{+}-u_{+}<v_{m}-u_{m}$.

In this case, we are concentrated on the interaction of $\delta S$ with $\overleftarrow{R}+J$ (see Figure 5). The state between $\overleftarrow{R}$ and $J$ is $\left(u_{1}, v_{1}\right)=\left(\left(v_{+}-u_{+}\right) u_{m} /\left(v_{m}-u_{m}\right),\left(v_{+}-u_{+}\right) v_{m} /\left(v_{m}-\underline{u}_{m}\right)\right)$. The propagation speeds of $\delta S_{1}$ and the wave back of $R$ are $\sigma_{1}=1 /\left(\left(1-u_{-}+v_{-}\right)\left(1-u_{m}+v_{m}\right)\right)$ and $\lambda_{1}\left(u_{m}, v_{m}\right)=1 /$ $\left(1-u_{m}+v_{m}\right)^{2}$. If $-1<v_{-}-u_{-}<0<v_{m}-u_{m}$, then the $\delta S$ will meet $R$ at point

$$
\left(x_{1}, t_{1}\right)=\left(\frac{1-u_{m}+v_{m}}{v_{m}-u_{m}-\left(v_{-}-u_{-}\right)}, \frac{\left(1-u_{-}+v_{-}\right)\left(1-u_{m}+v_{m}\right)^{2}}{v_{m}-u_{m}-\left(v_{-}-u_{-}\right)}\right) .
$$

The strength of $\delta S_{1}$ is given by

$$
\beta_{1}(t)=\frac{u_{-} v_{m}-u_{m} v_{-}}{\left(1-u_{-}+v_{-}\right)\left(1-u_{m}+v_{m}\right)} t .
$$

By substituting (37) into (38), we can obtain the following equation:

$$
\beta_{1}\left(t_{1}\right)=\frac{\left(u_{-} v_{m}-u_{m} v_{-}\right)\left(1-u_{m}+v_{m}\right)}{v_{m}-u_{m}-\left(v_{-}-u_{-}\right)} .
$$

After the time $t_{1}$, there is $\delta S_{2}$ which has the same expression of (28).

Next, we compute the strength of $\delta S_{2}$, by virtue of splitting the delta function method introduced by Nedeljkov and Oberguggeenberger in [35]. This method has also been used in $[36,37]$. Now, we construct the delta shock wave $x=x(t)$ which satisfies the following equation:

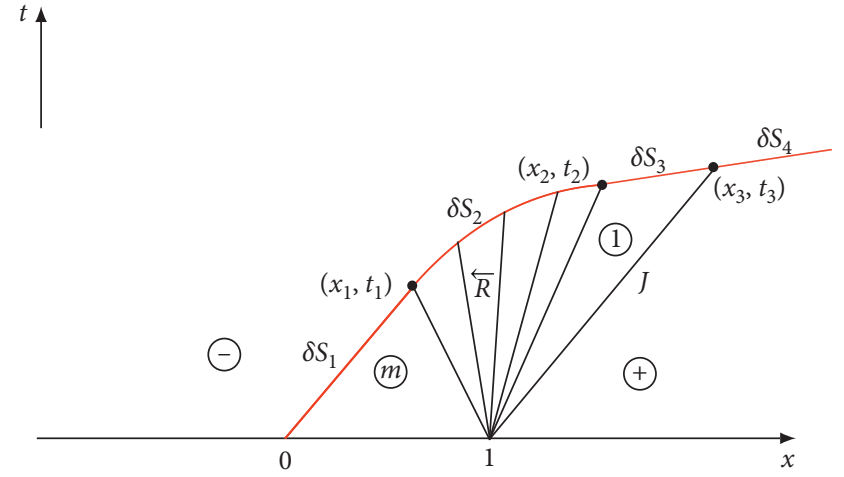

Figure 5: When $-1<v_{-}-u_{-}<0<v_{+}-u_{+}<v_{m}-u_{m}$.

$$
\begin{aligned}
& v(x, t)=\left\{\begin{array}{c}
v_{-}, \quad x<x(t), \\
\left(\sqrt{\frac{t}{x-1}}-1\right) \frac{v_{m}}{v_{m}-u_{m}}, \quad x>x(t)
\end{array}\right\} \\
& +\beta_{2}^{-}(t) \delta^{-}(x-x(t))+\beta_{2}^{+}(t) \delta^{+}(x-x(t)), \\
& u(x, t)=\left\{\begin{array}{c}
u_{-}, \quad x<x(t), \\
\left(\sqrt{\frac{t}{x-1}}-1\right) \frac{u_{m}}{v_{m}-u_{m}}, \quad x>x(t),
\end{array}\right\} \\
& +\beta_{2}^{-}(t) \delta^{-}(x-x(t))+\beta_{2}^{+}(t) \delta^{+}(x-x(t)) \text {, }
\end{aligned}
$$

in which $\quad \beta_{2}(t) \delta(x-x(t))=\beta_{2}^{-}(t) \delta^{-}(x-x(t))+\beta_{2}^{+}$ $(t) \delta^{+}(x-x(t))$ is a split delta function and $\beta_{2}(t)=\beta_{2}^{-}(t)+$ $\beta_{2}^{+}(t)$ denotes the strength of $\delta S_{2}$. Furthermore, we see from (40) and (41) that

$$
\begin{aligned}
v_{t}= & \frac{v_{m}}{v_{m}-u_{m}} \cdot \frac{H}{2 \sqrt{(x-1) t}}-\left(\left(\sqrt{\frac{t}{x-1}}-1\right) \frac{v_{m}}{v_{m}-u_{m}}-v_{-}\right) \\
& \cdot \delta \sigma_{2}+\left(\beta_{2}^{-{ }^{\prime}}(t)+\beta_{2}^{+^{\prime}}(t)\right) \delta-\sigma_{2}\left(\beta_{2}^{+}(t)+\beta_{2}^{-}(t)\right) \delta^{\prime}
\end{aligned}
$$

$$
\begin{aligned}
\left(\frac{v}{1-u+v}\right)_{x}= & -\frac{v_{m}}{v_{m}-u_{m}} \cdot \frac{H}{2 \sqrt{(x-1) t}} \\
& +\left(\frac{v_{m}}{v_{m}-u_{m}}-\frac{v_{m}}{v_{m}-u_{m}} \cdot \sqrt{\frac{x-1}{t}}-\frac{v_{-}}{1-u_{-}+v_{-}}\right) \delta \\
& +\left(\beta_{2}^{+}(t)+\beta_{2}^{-}(t)\right) \delta^{\prime}
\end{aligned}
$$

where $H, \delta$, and $\delta^{\prime}$ are the functions of $x-x(t)$; besides, $x(t)$ is given by (28). We substitute (42) and (43) into the first equation of (2) and compare the coefficients of $\delta$ and $\delta^{\prime}$, we obtain 


$$
\begin{gathered}
-\sigma_{2}\left(\left(\sqrt{\frac{t}{x-1}-1}\right) \frac{v_{m}}{v_{m}-u_{m}}-v_{-}\right)+\beta_{2}^{-^{\prime}}(t)+\beta_{2}^{+^{\prime}}(t) \\
+\frac{v_{m}}{v_{m}-u_{m}}\left(1-\sqrt{\frac{x-1}{t}}\right)-\frac{v_{-}}{1-u_{-}+v_{-}}=0 \\
-\sigma_{2}\left(\beta_{2}^{+}(t)+\beta_{2}^{-}(t)\right)+\beta_{2}^{+}(t)+\beta_{2}^{-}(t)=0 .
\end{gathered}
$$

According to (28), we have

$$
\sigma_{2}=\frac{1}{\left(1-u_{-}+v_{-}\right)(1-u+v)}=\frac{1}{1-u_{-}+v_{-}} \cdot \sqrt{\frac{x-1}{t}} .
$$

Replacing $\sigma_{2}$ by (46) in (44), we conclude that

$$
\begin{aligned}
\beta_{2}^{-}(t)+\beta_{2}^{+^{\prime}}(t)= & \left(\frac{v_{-}-u_{-}}{1-u_{-}+v_{-}}+\sqrt{\frac{v_{m}-u_{m}-\left(v_{-}-u_{-}\right)}{\left(1-u_{-}+v_{-}\right) t}}\right) \\
& \cdot \frac{u_{-} v_{m}-v_{-} u_{m}}{\left(1-u_{-}+v_{-}\right)\left(v_{m}-u_{m}\right)} .
\end{aligned}
$$

Integrating (47) with respect to $t$, recall the initial condition (39), thus

$$
\begin{aligned}
\beta_{2}(t)= & \frac{u_{-} v_{m}-v_{-} u_{m}}{\left(1-u_{-}+v_{-}\right)\left(v_{m}-u_{m}\right)} \\
& \cdot\left(\frac{v_{-}-u_{-}}{1-u_{-}+v_{-}} t+2 \sqrt{\frac{v_{m}-u_{m}-\left(v_{-}-u_{-}\right)}{1-u_{-}+v_{-}}} t-1\right. \\
& \left.+u_{m}-v_{m}\right) .
\end{aligned}
$$

Since $-1<v_{-}-u_{-}<0<v_{1}-u_{1}$, then the delta shock wave $\delta S_{2}$ will cross the whole $\overleftarrow{R}$ at the point

$$
\begin{aligned}
\left(x_{2}, t_{2}\right)= & \left(1+\frac{\left(v_{m}-u_{m}-v_{-}+u_{-}\right)\left(1-u_{-}+v_{-}\right)}{\left(v_{+}-u_{+}-v_{-}+u_{-}\right)^{2}},\right. \\
& \left.\frac{\left(v_{m}-u_{m}-v_{-}+u_{-}\right)\left(1-u_{-}+v_{-}\right)\left(1-u_{+}+v_{+}\right)}{\left(v_{+}-u_{+}-v_{-}+u_{-}\right)^{2}}\right) .
\end{aligned}
$$

After $t_{2}$, there is a new delta shock with $\delta S_{3}$ whose propagation velocity and strength, respectively, are given by

$$
\begin{aligned}
\sigma_{3} & =\frac{1}{\left(1-u_{-}+v_{-}\right)\left(1-u_{+}+v_{+}\right)} \\
\beta_{3}(t) & =\beta_{2}\left(t_{2}\right)+\frac{v_{+}-u_{+}}{v_{m}-u_{m}} \cdot \frac{u_{-} v_{m}-u_{m} v_{-}}{\left(1-u_{-}+v_{-}\right)\left(1-u_{+}+v_{+}\right)}\left(t-t_{2}\right),
\end{aligned}
$$

where $\beta_{2}\left(t_{2}\right)$ can be calculated by (48).
The $\delta S_{3}$ interacts with $J$ at point $\left(x_{3}, t_{3}\right)$ which is resolved by the following equation:

$$
\left\{\begin{array}{l}
x_{3}-1=\frac{t_{3}}{1-u_{+}+v_{+}}, \\
x_{3}-x_{2}=\frac{t_{3}-t_{2}}{\left(1-u_{-}+v_{-}\right)\left(1-u_{+}+v_{+}\right)} .
\end{array}\right.
$$

Recalling (49), the following is clear:

$$
\begin{aligned}
\left(x_{3}, t_{3}\right)= & \left(1+\frac{\left(v_{m}-u_{m}-v_{-}+u_{-}\right)\left(1-u_{-}+v_{-}\right)}{\left(v_{+}-u_{+}-v_{-}+u_{-}\right)\left(u_{-}-v_{-}\right)}\right. \\
& \left.\frac{\left(v_{m}-u_{m}-v_{-}+u_{-}\right)\left(1-u_{+}+v_{+}\right)\left(1-u_{-}+v_{-}\right)}{\left(v_{+}-u_{+}-v_{-}+u_{-}\right)\left(u_{-}-v_{-}\right)}\right) .
\end{aligned}
$$

After $t_{3}$, there is a new delta shock wave $\delta S_{4}$ which has the same speed with $\delta S_{3}$. The strength of $\delta S_{4}$ is given by

$$
\beta_{4}(t)=\beta_{3}\left(t_{3}\right)+\frac{u_{-} v_{+}-u_{+} v_{-}}{\left(1-u_{-}+v_{-}\right)\left(1-u_{+}+v_{+}\right)}\left(t-t_{3}\right), \quad t>t_{3} .
$$

Passing to limits as $\left(v_{-}-u_{-}\right) \longrightarrow(-1)^{+}$in (26), (49), and (53), we derive

$$
\begin{aligned}
\lim _{\left(v_{-}-u_{-}\right) \longrightarrow(-1)^{+}}\left(x_{1}, t_{1}\right) & =\lim _{\left(v_{-}-u_{-}\right) \longrightarrow(-1)^{+}}\left(x_{2}, t_{2}\right) \\
& =\lim _{\left(v_{-}-u_{-}\right) \longrightarrow(-1)^{+}}\left(x_{2}, t_{2}\right)=(1,0) .
\end{aligned}
$$

Since $\left(v_{-}-u_{-}\right) \longrightarrow(-1)^{+}$, then all the propagation speeds and strength of each delta shock wave tend to infinite. Thus, the solution of this case has a zero delta shock wave for $\left(v_{-}-u_{-}\right) \longrightarrow(-1)^{+}$.

Case 5. $-1<v_{-}-u_{-}<v_{+}-u_{+}<0<v_{m}-u_{m}$.

Based on the analysis of Case $4, \delta S_{1}$ overtakes $\overleftarrow{R}$ (see Figure 6). The intersection point $\left(x_{1}, t_{1}\right)$ and the strength of delta shock wave at $t_{1}$ are given by (26) and (39), respectively. The $\delta S_{2}$ satisfies (28) and its strength is given by (48). The interaction point of the curve of $\delta S_{2}$ and forward wave of $\overleftarrow{R}$ (or back wave of $\vec{R}$ ) can be calculated by the following equation:

$$
\left\{\begin{array}{l}
\sqrt{x_{2}-1}=\frac{\sqrt{t_{2}}}{1-u_{-}+v_{-}}-\sqrt{\frac{v_{m}-u_{m}-\left(v_{-}-u_{-}\right)}{1-u_{-}+v_{-}}}, \\
x_{2}-1=t_{2},
\end{array}\right.
$$

It follows from (56) that

$$
\begin{aligned}
\left(x_{2}, t_{2}\right)= & \left(1+\frac{\left(v_{m}-u_{m}-v_{-}+u_{-}\right)\left(1-u_{-}+v_{-}\right)}{\left(u_{-}-v_{-}\right)^{2}},\right. \\
& \left.\frac{\left(v_{m}-u_{m}-v_{-}+u_{-}\right)\left(1-u_{-}+v_{-}\right)}{\left(u_{-}-v_{-}\right)^{2}}\right) .
\end{aligned}
$$




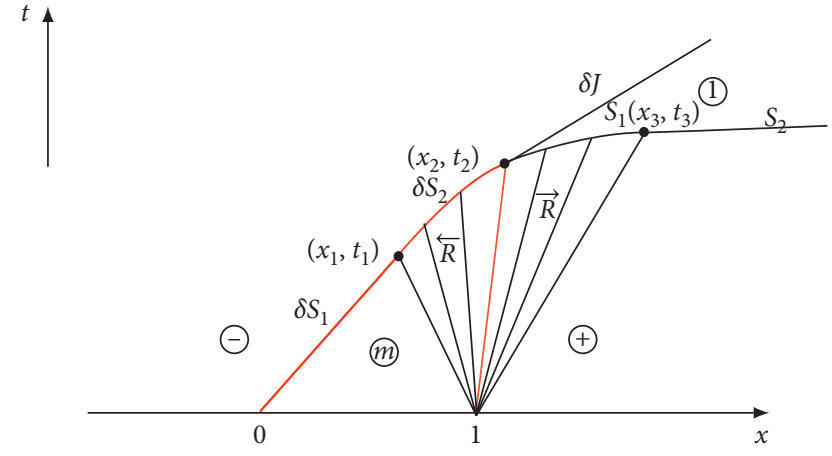

FIgURE 6: When $-1<v_{-}-u_{-}<v_{+}-u_{+}<0<v_{m}-u_{m}$.

In view of (48) and (57), we conclude

$$
\beta_{2}\left(t_{2}\right)=\frac{u_{-} v_{m}-v_{-} u_{m}}{u_{-}-v_{-}}
$$

It is easy to see that the state $(u, v)$ of $\vec{R}$ satisfies $u / u_{+}=v / v_{+}$. At the point $\left(x_{2}, t_{2}\right)$, a new local Riemann problem containing a Dirac delta measure is formulated as $\left.v\right|_{t=t_{2}}=\left\{\begin{array}{cc}v_{-}, & x<x_{2} \\ \theta, & x>x_{2}\end{array}\right\}+\beta_{2}\left(t_{2}\right) \delta\left(x-x_{2}-\frac{t-t_{2}}{1-u_{-}+v_{-}}\right)$,

$\left.u\right|_{t=t_{2}}=\left\{\begin{array}{cc}u_{-}, & x<x_{2} \\ \frac{\theta u_{+}}{v_{+}}, & x>x_{2}\end{array}\right\}+\beta_{2}\left(t_{2}\right) \delta\left(x-x_{2}-\frac{t-t_{2}}{1-u_{-}+v_{-}}\right)$

here we assume that $\theta \longrightarrow 0$ and satisfies $(x-1) / t=1 /(1+\theta)^{2}$.

At the time $t_{2}$, the initial data become (59) and (60), we construct the solution with the following form:

$$
\begin{aligned}
v= & \left\{\begin{array}{cc}
v_{-}, & x<x_{2}, \\
\frac{v_{-}-u_{-}}{v_{+}-u_{+}} v_{+}, & x_{2}<x<x(t), \\
\theta, & x>x(t),
\end{array}\right\} \\
& +\beta_{2}\left(t_{2}\right) \delta\left(x-x_{2}-\frac{t-t_{2}}{1-u_{-}+v_{-}}\right), \\
u= & \left\{\begin{array}{cc}
\frac{v_{-}-u_{-}}{v_{+}-u_{+}}, & x<x_{2}, \\
\frac{\theta u_{+}}{v_{+}}, & x>x(t),
\end{array}\right\} \\
& +\beta_{2}\left(t_{2}\right) \delta\left(x-x_{2}-\frac{t-t_{2}}{1-u_{-}+v_{-}}\right),
\end{aligned}
$$

where $x(t)=x_{2}+t-t_{2} /\left(\left(1-u_{-}+v_{-}\right)\left(1-\left(\theta u_{+} / v_{+}\right)+\theta\right)\right)$ given nearby $\left(x_{2}, t_{2}\right)$ for sufficiently small $\theta$ denotes shock wave curve of $S_{1}$.
Next, we clarify equalities (61) and (62) satisfy equations (2) with initial data (59) and (60) in the weak sense. Combining (61) and (62), we deduce

$$
\begin{aligned}
v_{t}= & \left(\frac{v_{-}-u_{-}}{v_{+}-u_{+}} v_{+}\right) \delta \cdot\left(-\frac{1}{1-u_{-}+v_{-}}\right) \\
& +\beta_{2}\left(t_{2}\right) \delta^{\prime}\left(-\frac{1}{1-u_{-}+v_{-}}\right) \\
\left(\frac{v}{1-u+v}\right)_{x}= & \left(\frac{\left(v_{-}-u_{-}\right) v_{+}}{\left(1-u_{-}+v_{-}\right)\left(v_{+}-u_{+}\right)}-\frac{v_{-}}{1-u_{-}+v_{-}}\right) \delta \\
& +\frac{\beta_{2}\left(t_{2}\right)}{1-u_{-}+v_{-}} \delta^{\prime},
\end{aligned}
$$

where $\delta$ and $\delta^{\prime}$ are the functions of $x-x_{2}-\left(t-t_{2}\right) /\left(1-u_{-}+v_{-}\right)$. In light of (63) and (64), we thus deduce that

$$
v_{t}+\left(\frac{v}{1-u+v}\right)_{x}=0 .
$$

Similarly, we claim that

$$
u_{t}+\left(\frac{u}{1-u+v}\right)_{x}=0
$$

Since contact discontinuity line $x=x_{2}+\left(t-t_{2}\right) /(1-$ $\left.u_{-}+v_{-}\right)$with a Dirac delta function, then there is a delta contact discontinuity $\delta J$ in [35]. After $t_{2}, \delta S_{2}$ splits into $\delta J$ and $S_{1}$ which are connected by intermediate state $\left(u_{1}, v_{1}\right)=\left(\left(v_{-}-u_{-} / v_{+}-u_{+}\right) v_{+},\left(v_{-}-u_{-} / v_{+}-u_{+}\right) u_{+}\right)$. The $\delta J$ continues to move forwards with the constant speed $\tau=$ $1 /\left(1-u_{-}+v_{-}\right)$and the strength $\beta_{2}\left(t_{2}\right)$ is given by (58). Besides, $S_{1}$ continues to cross the $\vec{R}$ with the initial speed $\omega_{1}=1 /\left(1-u_{-}+v_{-}\right)$. During the process, the state of the left-hand side is $\left(u_{1}, v_{1}\right)$ and the right-hand side is $(u, v)=$ $\left((\sqrt{(t / x-1)}-1) u_{+} / v_{+}-u_{+},(\sqrt{(t / x-1)}-1) v_{+} / v_{+}-u_{+}\right)$. In the physics plane, the curve of $S_{1}$ satisfies the following equation:

$$
\sqrt{x-1}=\frac{\sqrt{t}}{1-u_{-}+v_{-}}-\sqrt{\frac{v_{m}-u_{m}-v_{-}+u_{-}}{1-u_{-}+v_{-}}} .
$$

According to $-1 \leq v_{-}-u_{-}<v_{+}-u_{+}<0$, the $S_{1}$ can penetrate the whole $\vec{R}$ completely at the point $\left(x_{3}, t_{3}\right)$, which is

$$
\begin{aligned}
\left(x_{3}, t_{3}\right)= & \left(1+\frac{\left(v_{m}-u_{m}-v_{-}+u_{-}\right)\left(1-u_{-}+v_{-}\right)}{\left(v_{+}-u_{+}-v_{-}+u_{-}\right)^{2}},\right. \\
& \left.\frac{\left(v_{m}-u_{m}-v_{-}+u_{-}\right)\left(1-u_{-}+v_{-}\right)\left(1-u_{+}+v_{+}\right)^{2}}{\left(v_{+}-u_{+}-v_{-}+u_{-}\right)^{2}}\right) .
\end{aligned}
$$

After $t_{3}$, the shock wave is denoted by $S_{2}$ which has the propagation speed $\omega_{2}=1 /\left(\left(1-u_{-}+v_{-}\right)\left(1-u_{+}+v_{+}\right)\right)$.

As $\left(v_{-}, u_{-}\right) \longrightarrow(-1)^{+}$, it is clear to see that all intersection points tend to $(1,0)$ and all speeds of waves tend to 
infinite. Thus, a zero delta shock will appear in the interval $[0,1]$. For $x>1$, the zero delta shock wave also emerges since the superposition of a zero delta contact discontinuity and a zero shock wave.

Case 6. $-1<v_{-}-u_{-}<0<v_{m}-u_{m}<v_{+}-u_{+}$.

In this case, we consider the interaction of $\delta S$ with $\overleftarrow{S}+J$ and the middle state of $\overleftarrow{S}$ and $J$ is $\left(u_{1}, v_{1}\right)=\left(\left(v_{+}-u_{+}\right) u_{m} /\right.$ $\left.v_{m}-u_{m},\left(v_{+}-u_{+}\right) v_{m} / v_{m}-u_{m}\right)$. It is clear to see that $\delta S_{1}$ catches up $\overleftarrow{S}$ in finite time, and the intersection point $\left(x_{1}, t_{1}\right)$ can be seen in (33) (see Figure 7). Like as before, due to $-1<v_{-}-u_{-}<0<v_{+}-u_{+}$, after the interaction of $\delta S_{1}$ and $\overleftarrow{S}$, there is a new delta shock wave $\delta S_{2}$ whose propagation speed is $\sigma_{2}=1 /\left(\left(1-u_{-}+v_{-}\right)\left(1-u_{+}+v_{+}\right)\right)$.

For $\sigma_{2}>\tau_{1}=1 /\left(1-u_{+}+v_{+}\right), \delta S_{2}$ interacts with $J_{1}$ at the point $\left(x_{2}, t_{2}\right)$ which satisfies the following equation:

$$
\left\{\begin{array}{l}
x_{2}-x_{1}=\frac{t_{2}-t_{1}}{\left(1-u_{-}+v_{-}\right)\left(1-u_{+}+v_{+}\right)}, \\
x_{2}-1=\frac{t_{2}}{1-u_{+}+v_{+}} .
\end{array}\right.
$$

Together with (33), we deduce that

$$
\begin{aligned}
\left(x_{2}, t_{2}\right)= & \left(\frac{\left(v_{-}-u_{-}-v_{m}+u_{m}\right)\left(1-u_{-}+v_{-}\right)}{\left(v_{+}-u_{+}-v_{-}+u_{-}\right)\left(v_{-}-u_{-}\right)}+1,\right. \\
& \left.\frac{\left(v_{-}-u_{-}-v_{m}+u_{m}\right)\left(1-u_{-}+v_{-}\right)\left(1-u_{+}+v_{+}\right)}{\left(v_{+}-u_{+}-v_{-}+u_{-}\right)\left(v_{-}-u_{-}\right)}\right) .
\end{aligned}
$$

When the delta shock wave passes through $J_{1}$, it continues to move forward without change of direction. The strengths of delta shock waves are given by

$$
\begin{gathered}
\beta_{1}(t)=\frac{u_{-} v_{m}-u_{m} v_{-}}{\left(1-u_{-}+v_{-}\right)\left(1-u_{m}+v_{m}\right)} t, \quad 0 \leq t \leq t_{1}, \\
\beta_{2}(t)=\beta_{1}\left(t_{1}\right)+\frac{u_{-} v_{1}-u_{1} v_{-}}{\left(1-u_{-}+v_{-}\right)\left(1-u_{1}+v_{1}\right)}\left(t-t_{1}\right), \quad t_{1} \leq t \leq t_{2}, \\
\beta_{3}(t)=\beta_{2}\left(t_{2}\right)+\frac{u_{-} v_{+}-u_{+} v_{-}}{\left(1-u_{-}+v_{-}\right)\left(1-u_{+}+v_{+}\right)}\left(t-t_{2}\right), \quad t \geq t_{2},
\end{gathered}
$$

in which $t_{1}$ and $t_{2}$ can be obtained by (33) and (71).

If $\left(v_{-}-u_{-}\right) \longrightarrow(-1)^{+}$, then the propagation speeds and strengths of all delta shock tend to infinite. Therefore, there is a zero delta shock in the positive $x$-axis as $\left(v_{-}-u_{-}\right) \longrightarrow(-1)^{+}$.

In this section, we mainly testify the type (2) of Theorem 1.

\section{Analysis of Zero Rarefaction Wave}

In this section, we are interested in the zero rarefaction wave interacting with the other waves. As $-1<v_{m}-u_{m}<-1+\varepsilon$, for sufficiently small $\varepsilon$ and $\left(v_{m}-u_{m}\right) \longrightarrow(-1)^{+}$, there is a zero rarefaction wave at the point $(0,0)$.

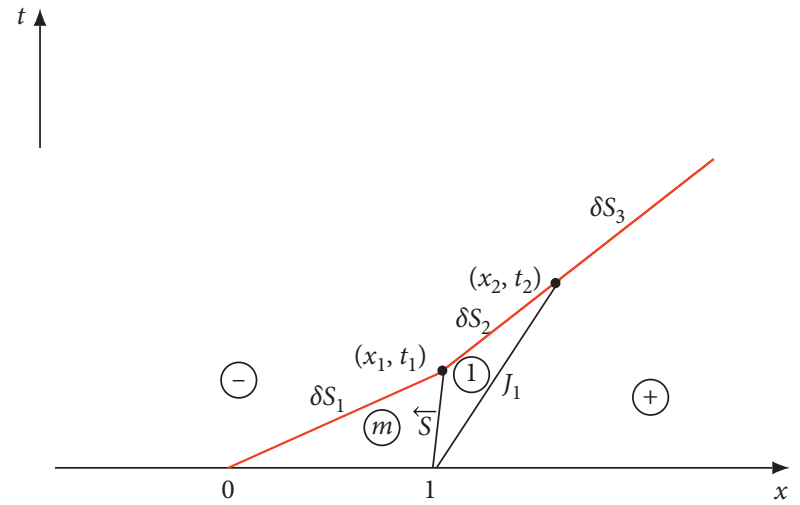

FIGURE 7: When $-1<v_{-}-u_{-}<0<v_{m}-u_{m}<v_{+}-u_{+}$.

According to $v_{-}-u_{-}<0$ or not, we separate our discussion into two parts. To be more precise, if $v_{-}-u_{-}<0$ and $-1<v_{m}-u_{m}<-1+\varepsilon$, for sufficiently small $\varepsilon$, then the Riemann solution of (2) at the point $(0,0)$ is $J+\vec{R}$. Otherwise, the Riemann solution is $\overleftarrow{R}+\vec{R}$ at the point $(0,0)$ Firstly, we study the $v_{-}-u_{-}>0$ and then consider the situation $v_{-}-u_{-}<0$.

Case 7. $-1<v_{m}-u_{m}<v_{-}-u_{-}<v_{+}-u_{+}<0$.

When $-1<v_{m}-u_{m}<v_{-}-u_{-}<v_{+}-u_{+}<0$ (see Figure 8$)$, the middle state satisfies $\left(u_{1}, v_{1}\right)=\left(\left(v_{-}-u_{-} / v_{m}-\right.\right.$ $\left.\left.u_{m}\right) u_{m},\left(v_{-}-u_{-} / v_{m}-u_{m}\right) v_{m}\right)$ and $\left(u_{2}, v_{2}\right)=\left(\left(v_{m}-u_{m} /\right.\right.$ $\left.\left.v_{+}-u_{+}\right) u_{+},\left(v_{m}-u_{m} / v_{+}-u_{+}\right) v_{+}\right)$, respectively. The propagation speeds of $J_{2}$ and the wave front of $\overrightarrow{R_{1}}$ are $\tau_{2}=$ $1 /\left(1-u_{m}+v_{m}\right) \quad$ and $\quad \lambda_{2}^{1}\left(u_{m}, v_{m}\right)=1 /\left(1-u_{m}+v_{m}\right)^{2}$, respectively.

It is easy to see that $\vec{R}$ overtakes $J_{2}$ in finite time, and the interaction point is

$$
\left(x_{1}, t_{1}\right)=\left(\frac{1}{u_{m}-v_{m}}, \frac{\left(1-u_{m}+v_{m}\right)^{2}}{u_{m}-v_{m}}\right) .
$$

Based on the above discussions, we derive that $J_{2}$ begins to penetrate $\overrightarrow{R_{1}}$ at point $\left(x_{1}, t_{1}\right)$. During the process of penetration, there is a new contract discontinuity $J_{3}$ satisfying the following equation:

$$
\sqrt{x}=\sqrt{t}+\sqrt{u_{m}-v_{m}} .
$$

When $J_{3}$ crosses the whole $\overrightarrow{R_{1}}$, there is a new wave $J_{4}$ whose propagation speed is same with $J_{1}$.

Besides, we denote $\overrightarrow{R_{2}}$ after $\overrightarrow{R_{1}}$ penetrates $J_{3}$. The $\overrightarrow{R_{2}}$ has the same propagation speed with $\overrightarrow{R_{1}}$. The shock $\overrightarrow{S_{2}}$ will penetrate $\overrightarrow{R_{2}}$, and the new shock $\overrightarrow{S_{2}}$ appears after $t_{2}$. The wave $\overrightarrow{S_{2}}$ penetrates whole $\overrightarrow{R_{2}}$ at the point $\left(x_{3}, t_{3}\right)$ :

$$
\begin{aligned}
\left(x_{3}, t_{3}\right)= & \left(\frac{\left(v_{+}-u_{+}-v_{m}+u_{m}\right)\left(1-u_{+}+v_{+}\right)}{\left(v_{+}-u_{+}-v_{-}+u_{-}\right)^{2}}\right. \\
& \left.\frac{\left(v_{+}-u_{+}-v_{m}+u_{m}\right)\left(1-u_{+}+v_{+}\right)\left(1-u_{-}+v_{-}\right)^{2}}{\left(v_{+}-u_{+}-v_{-}+u_{-}\right)^{2}}\right) .
\end{aligned}
$$




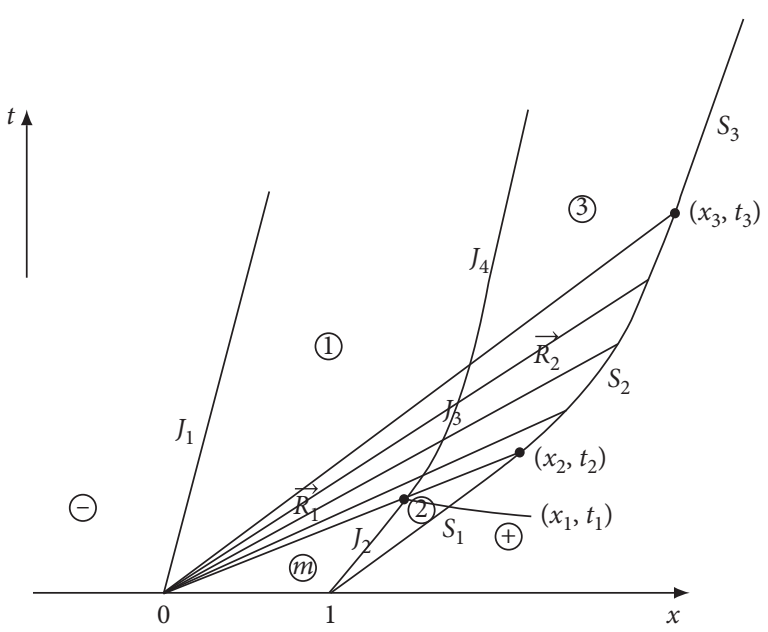

Figure 8: When $-1<v_{m}-u_{m}<v_{-}-u_{-}<v_{+}-u_{+}<0$.

After $t_{3}$, shock wave $S_{2}$ turns into $S_{3}$ with a speed $\omega_{3}=1 /\left(\left(1-u_{-}+v_{-}\right)\left(1-u_{+}+v_{+}\right)\right)$.

In the same way, for $\left(v_{m}-u_{m}\right) \longrightarrow(-1)^{+}$, it is easy to deduce that there is a zero rarefaction wave in the interval $[0,1]$.

Case 8. $-1<v_{m}-u_{m}<v_{+}-u_{+}<v_{-}-u_{-}<0$.

In this case, it is similar to the analysis of Case 7 . The difference is that the shock wave cannot penetrate $\overrightarrow{R_{2}}$ completely.

Case 9. $-1<v_{m}-u_{m}<v_{-}-u_{-}<0<v_{+}-u_{+}$.

Similarly, Case 5 , for $\left(v_{m}-u_{m}\right) \longrightarrow(-1)^{+}, \delta S$ coincides with positive $x$-axis at the point $(1,0)$ and $\delta S$ penetrates $\vec{R}$ completely. Furthermore, if $\left(v_{m}-u_{m}\right) \longrightarrow(-1)^{+}$, the zero rarefaction wave occurs in the interval $[0,1]$.

Case 10. $-1<v_{m}-u_{m}<0<v_{-}-u_{-}<v_{+}-u_{+}$.

From Figure 9, the delta shock wave interaction $\vec{R}$ at the point is as follows:

$$
\left(x_{1}, t_{1}\right)=\left(\frac{1-u_{+}+v_{+}}{v_{+}-u_{+}-v_{m}+u_{m}}, \frac{\left(1-u_{m}+v_{m}\right)^{2}\left(1-u_{+}+v_{+}\right)}{v_{+}-u_{+}-v_{m}+u_{m}}\right) \text {. }
$$

The strength of $\delta S_{1}$ at the $t_{1}$ is given by

$$
\beta_{1}\left(t_{1}\right)=\frac{\left(u_{m} v_{+}-u_{+} v_{m}\right)\left(1-u_{m}+v_{m}\right)}{v_{+}-u_{+}-v_{m}+u_{m}} .
$$

When $t>t_{1}$, a new delta shock wave $\delta S_{2}$ is generated satisfying

$$
\sqrt{x}=\frac{\sqrt{t}}{1-u_{+}+v_{+}}+\sqrt{\frac{v_{+}-u_{+}-v_{m}+u_{m}}{1-u_{+}+v_{+}}} .
$$

The $\delta S_{2}$ penetrates $\vec{R}$ completely at the point

$$
\begin{aligned}
\left(x_{2}, t_{2}\right)= & \left(\frac{\left(v_{+}-u_{+}-v_{m}+u_{m}\right)\left(1-u_{+}+v_{+}\right)}{v_{+}-u_{+}},\right. \\
& \left.\frac{\left(v_{+}-u_{+}-v_{m}+u_{m}\right)\left(1-u_{+}+v_{+}\right)}{v_{+}-u_{+}}\right) .
\end{aligned}
$$

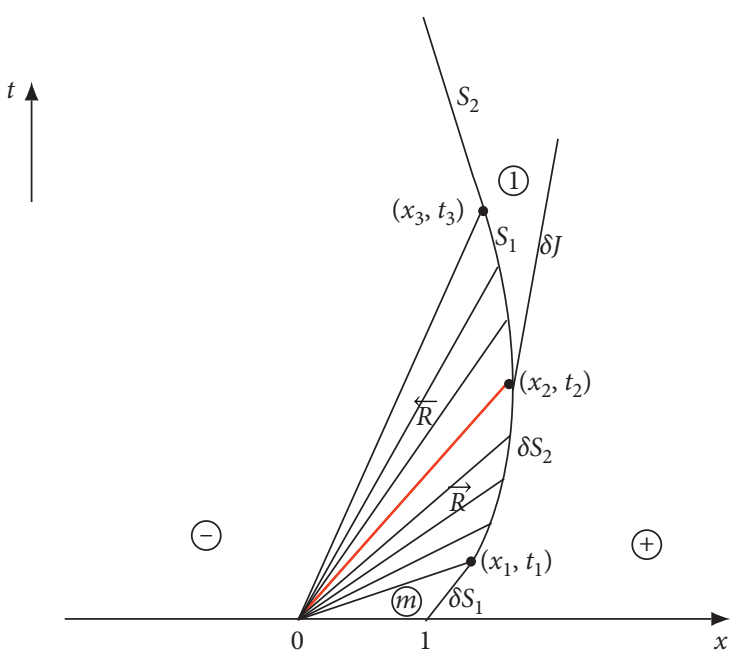

FIGURE 9: When $-1<v_{m}-u_{m}<0<v_{-}-u_{-}<v_{+}-u_{+}$.

As in Case 5, we construct the delta shock wave as follows:

$$
\begin{aligned}
v(x, t)= & \left\{\begin{array}{c}
\frac{v_{m}}{v_{m}-u_{m}}\left(\sqrt{\frac{t}{x}}-1\right), \quad x<x(t), \\
v_{+}, \quad x>x(t),
\end{array}\right\} \\
& +\beta_{2}^{-}(t) \delta^{-}(x-x(t))+\beta_{2}^{+}(t) \delta^{+}(x-x(t)), \\
u(x, t)= & \left\{\begin{array}{cc}
\frac{u_{m}}{v_{m}-u_{m}}\left(\sqrt{\frac{t}{x}}-1\right), \quad x<x(t), \\
u_{+}, \quad x>x(t),
\end{array}\right\} \\
& +\beta_{2}^{-}(t) \delta^{-}(x-x(t))+\beta_{2}^{+}(t) \delta^{+}(x-x(t)),
\end{aligned}
$$

where $x(t)$ is given by (79). Applying (81), (82) implies

$$
\begin{aligned}
v_{t}= & \frac{v_{m}}{v_{m}-u_{m}} \frac{1}{2 \sqrt{x t}}-\frac{v_{m}}{v_{m}-u_{m}} \frac{H}{2 \sqrt{x t}} \\
& -\left(v_{+}-\frac{v_{m}}{v_{m}-u_{m}}\left(\sqrt{\frac{t}{x}}-1\right)\right) \delta \sigma_{2}+\left(\beta_{2}^{-^{\prime}}(t)+\beta_{2}^{+^{\prime}}(t)\right) \delta \\
& -\left(\beta_{2}^{-}(t)+\beta_{2}^{+}(t)\right) \delta^{\prime} \sigma_{2},
\end{aligned}
$$

$$
\begin{aligned}
\left(\frac{v}{1-u+v}\right)_{x}= & -\frac{v_{m}}{v_{m}-u_{m}} \frac{1}{2 \sqrt{x t}}+\frac{v_{m}}{v_{m}-u_{m}} \frac{H}{2 \sqrt{x t}} \\
& +\left(\frac{v_{+}}{1-u_{+}+v_{+}}-\frac{v_{m}}{v_{m}-u_{m}}\left(1-\sqrt{\frac{x}{t}}\right)\right) \delta \\
& +\left(\beta_{2}^{-}(t)+\beta_{2}^{+}(t)\right) \delta^{\prime}
\end{aligned}
$$

where $\sigma_{2}$ is the propagation speed of $\delta S_{2}$. Substituting (83) and (84) into first equation of (2) and comparing the coefficients of $\delta$ and $\delta^{\prime}$, by virtue of (79), we obtain 


$$
\begin{aligned}
\beta_{2}^{\prime}(t)= & \beta_{2}^{-^{\prime}}(t)+\beta_{2}^{+^{\prime}}(t)=\frac{v_{+} u_{m}-v_{m} u_{+}}{\left(1-u_{+}+v_{+}\right)\left(v_{m}-u_{m}\right)} \\
& \cdot\left(\frac{v_{+}-u_{+}}{1-u_{+}+v_{+}}-\sqrt{\frac{v_{+}-u_{+}-v_{m}+u_{m}}{\left(1-u_{+}+v_{+}\right) t}}\right) .
\end{aligned}
$$

Recalling (78), we deduce that

$$
\begin{aligned}
\beta_{2}(t)= & \frac{v_{+} u_{m}-v_{m} u_{+}}{\left(1-u_{+}+v_{+}\right)\left(v_{m}-u_{m}\right)} \\
& \cdot\left(\frac{v_{+}-u_{+}}{1-u_{+}+v_{+}} t-2 \sqrt{\frac{v_{+}-u_{+}-v_{m}+u_{m}}{1-u_{+}+v_{+}}} t-1+u_{m}-v_{m}\right) .
\end{aligned}
$$

After the time $t_{2}, \delta S_{2}$ is decomposed into a shock wave $S_{1}$ and a delta contact discontinuity $\delta J$ with the middle state $\left(u_{1}, v_{1}\right)=\left(\left(v_{+}-u_{+} / v_{-}-u_{-}\right) u_{+},\left(v_{+}-u_{+} / v_{-}-u_{-}\right) v_{+}\right)$between them. Consequently, $\delta J$ continues to move forwards with the constant speed $1 /\left(1-u_{+}+v_{+}\right)$and the invariant strength $\beta_{2}\left(t_{2}\right)$. On the other hand, $S_{1}$ continues to penetrate $\overleftarrow{R}$ with a varying propagation speed, which satisfies the following equation:

$$
\left\{\begin{array}{l}
\frac{d x}{d t}=\frac{1}{1-u_{+}+v_{+}} \sqrt{\frac{x}{t}}, \\
\left(x_{2}, t_{2}\right)=\left(\frac{\left(v_{+}-u_{+}-v_{m}+u_{m}\right)\left(1-u_{+}+v_{+}\right)}{v_{+}-u_{+}},\right. \\
\left.\frac{\left(v_{+}-u_{+}-v_{m}+u_{m}\right)\left(1-u_{+}+v_{+}\right)}{v_{+}-u_{+}}\right),
\end{array}\right.
$$

thus

$$
\sqrt{x}=\frac{\sqrt{t}}{1-u_{+}+v_{+}}+\sqrt{\frac{\left(v_{+}-u_{+}-v_{m}+u_{m}\right)\left(v_{+}-u_{+}\right)}{1-u_{+}+v_{+}}} .
$$

For $-1<0<v_{+}-u_{+}<v_{-}-u_{-}, S_{1}$ is able to penetrate whole $\overleftarrow{R}$

For $\left(v_{m}-u_{m}\right) \longrightarrow(-1)^{+}$, it is clear to see that zero rarefaction wave only exists in the interval $[0,1]$ since the delta shock wave begins to penetrate the rarefaction wave from the point $(1,0)$.

Case 11. $-1<v_{m}-u_{m}<0<v_{+}-u_{+}<v_{-}-u_{-}$.

In this case, it is easy to find similar to Case 10 , and $S_{1}$ cannot penetrate $\overleftarrow{R}$ completely and has the asymptote line $x=t /\left(1-u_{+}+v_{+}\right)^{2}$.

Case 12. $-1<v_{m}-u_{m}<v_{+}-u_{+}<0<v_{-}-u_{-}$.

As in this case, we are concerned with the interaction of $\overleftarrow{R}+\vec{R}$ and $J+S$. In the beginning, the situation is similar to Case 7. More precisely, $J$ has asymptote $x=t$ and do not able to enter the region of $\overleftarrow{R}$. On the other hand, $\vec{R}$ intersects with $J$ denoted $\overrightarrow{R_{1}}$, and the shock wave $S$ cannot penetrate whole $\overrightarrow{R_{1}}$ and finally has the asymptote $x=t /\left(1-u_{+}+v_{+}\right)^{2}$.

Therefore, we have proved the type (3) of Theorem 1.

\section{Numerical Simulations}

In this section, we present some representative numerical simulations for the formation of zeros waves, mentioned in this paper, when the differences of initial data tend to -1 from the right side. In this paper, we employ the upwind scheme to validate our main results.

The following numerical simulations correspond to the zero shock wave of Case 1 (Figure 2). We take the initial data as follows:

$$
\begin{aligned}
& u_{-}=1.9, v_{-}=1, \\
& u_{m}=3.5, \\
& v_{m}=3, \\
& u_{+}=2.7, \\
& v_{+}=2, \\
& u_{-}=1.99, \\
& v_{-}=1, \\
& u_{m}=3.5, \\
& v_{m}=3, \\
& u_{+}=2.7, \\
& v_{+}=2 .
\end{aligned}
$$

This implies that the $v_{-}-u_{-}$from -0.9 (Figure 10) tends to -0.99 (Figure 11). It can be clearly seen from the numerical results that when $v_{-}-u_{-}$approaches -0.99 from $-0.9, u, v$ and the region of negative states all increase. Namely, the velocity of shock wave increases. That is to say, if $-1<v_{-}-u_{-}<v_{m}-u_{m}<0, v_{+}-u_{+}<0$ and if $\left(v_{-}-u_{-}\right) \longrightarrow$ $(-1)^{+}$, then the velocity tends to $\infty$. There is a zero shock wave.

The numerical simulations (Figures 12 and 13) correspond to the zero delta shock wave of Case 4 (Figure 5) with initial data as follows:

$$
\begin{aligned}
& u_{-}=1.9, \\
& v_{-}=1, \\
& u_{m}=1, \\
& v_{m}=3, \\
& u_{+}=50, \\
& v_{+}=51, \\
& u_{-}=1.99, \\
& v_{-}=1, \\
& u_{m}=1, \\
& v_{m}=3, \\
& u_{+}=50, \\
& v_{+}=51 .
\end{aligned}
$$




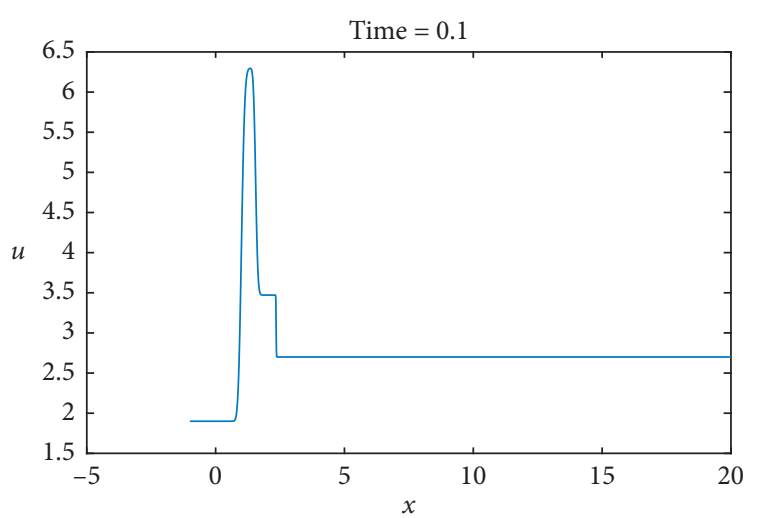

(a)

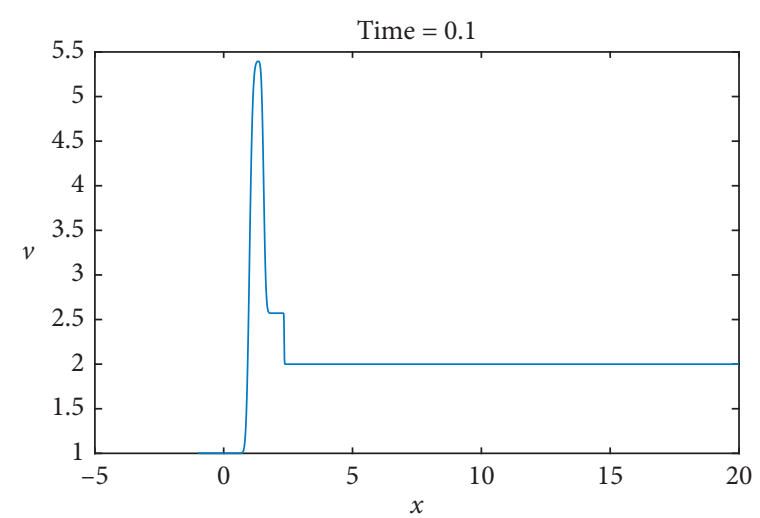

(b)

FIGURE 10: (a) $u$ and (b) $v$ for $v_{-}-u_{-}=0.9$ with $v_{-}=1, u_{-}=1.9, v_{m}=3.5, u_{m}=3, v_{+}=2$, and $u_{+}=2.7$.

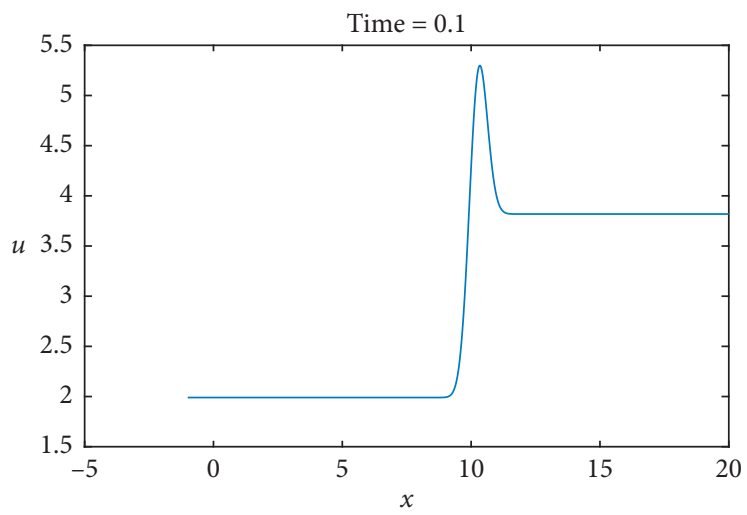

(a)

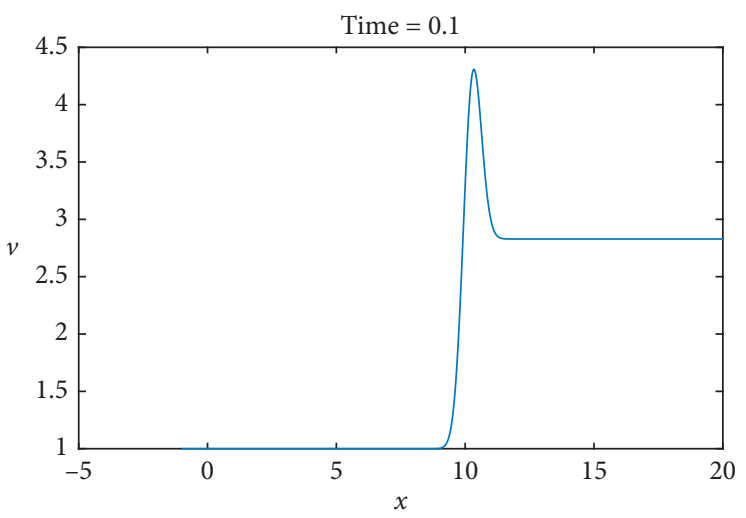

(b)

FIGURE 11: (a) $u$ and (b) $v$ for $v_{-}-u_{-}=0.99$ with $v_{-}=1, u_{-}=1.99, v_{m}=3.5, u_{m}=3, v_{+}=2$, and $u_{+}=2.7$.

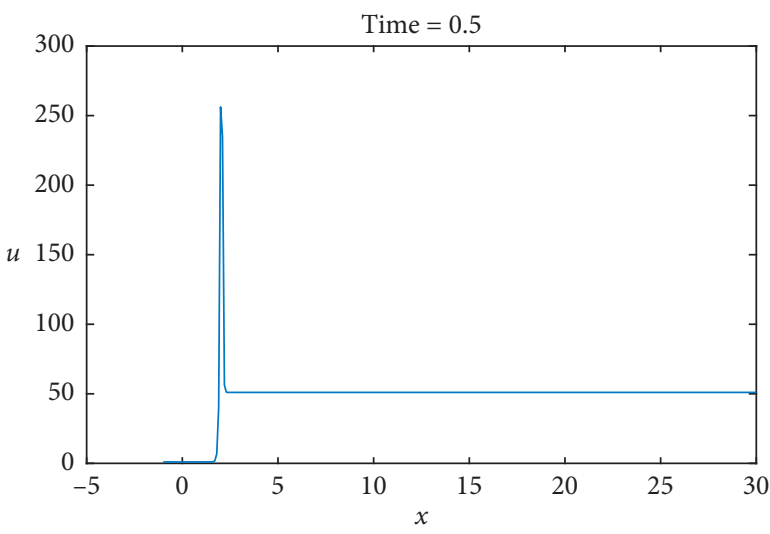

(a)

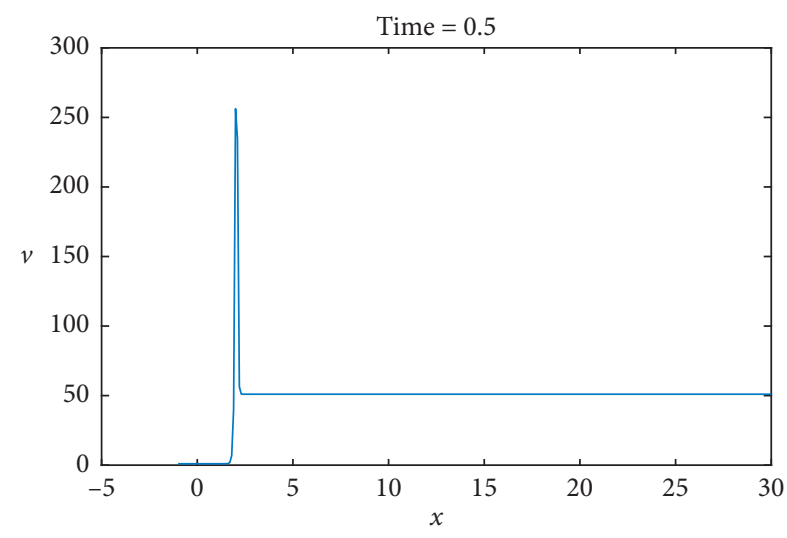

(b)

FIGURE 12: (a) $u$ and (b) $v$ for $v_{-}-u_{-}=0.9$ with $u_{-}=1.9, v_{-}=1, u_{m}=1, v_{m}=3, u_{+}=50$, and $v_{+}=51$.

That is to say, $v_{-}-u_{-}$from -0.9 (Figure 12 ) tends to -0.99 (Figure 13). As can be seen from the following numerical results, the negative state region and the values of $u$ and $v$ are getting more larger. As $v_{-}-u_{-}$tends to -1 , the shock velocity and $u, v$ approach $\infty$. In other words, when $-1<v_{-}-u_{-}<0<v_{m}-u_{m}, v_{-}-u_{-}<v_{+}-u_{+}$, and $\left(v_{-}-u_{-}\right) \longrightarrow$ $(-1)^{+}$, there is a zero delta shock wave.

The numerical simulations (Figures 14 and 15) correspond to the zero rarefaction waves of Case 7 (Figure 8) with the initial data as follows: 


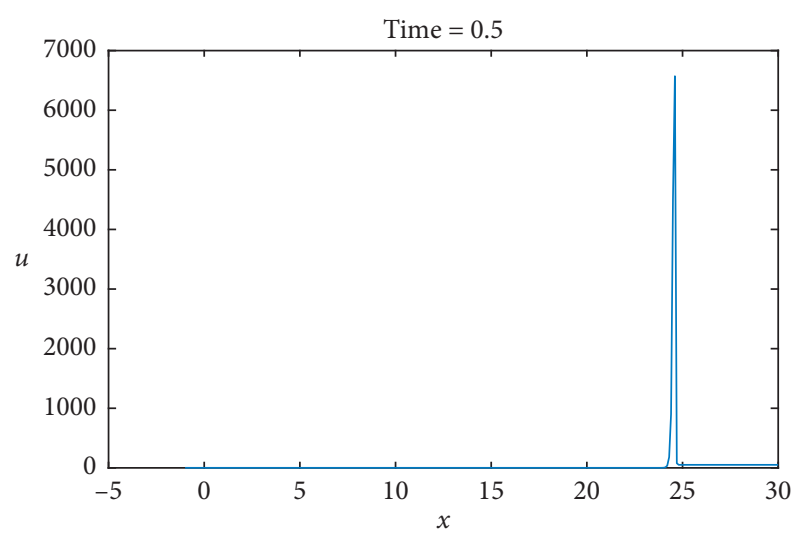

(a)

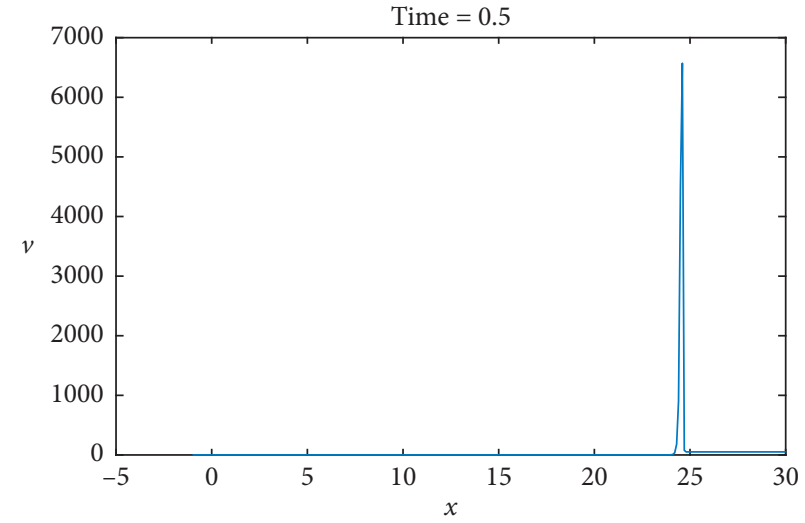

(b)

FIGURE 13: (a) $u$ and (b) $v$ for $v_{-}-u_{-}=0.99$ with $u_{-}=1.99, v_{-}=1, u_{m}=1, v_{m}=3, u_{+}=50$, and $v_{+}=51$.

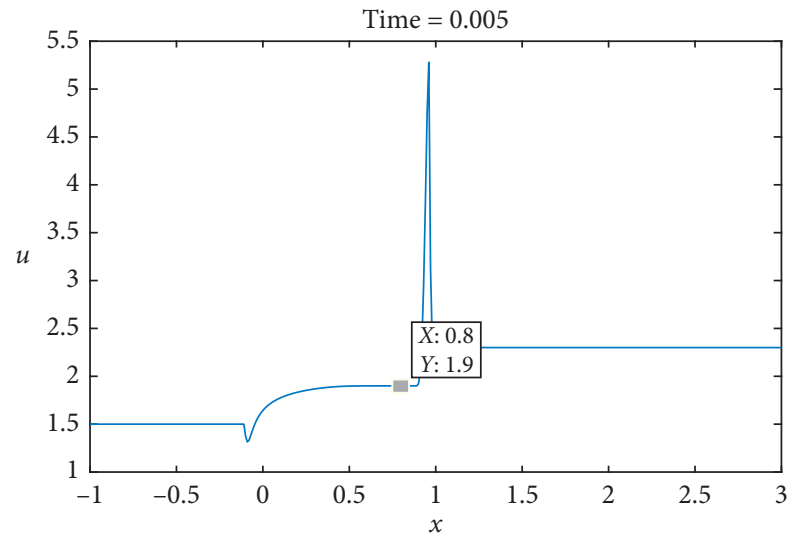

(a)

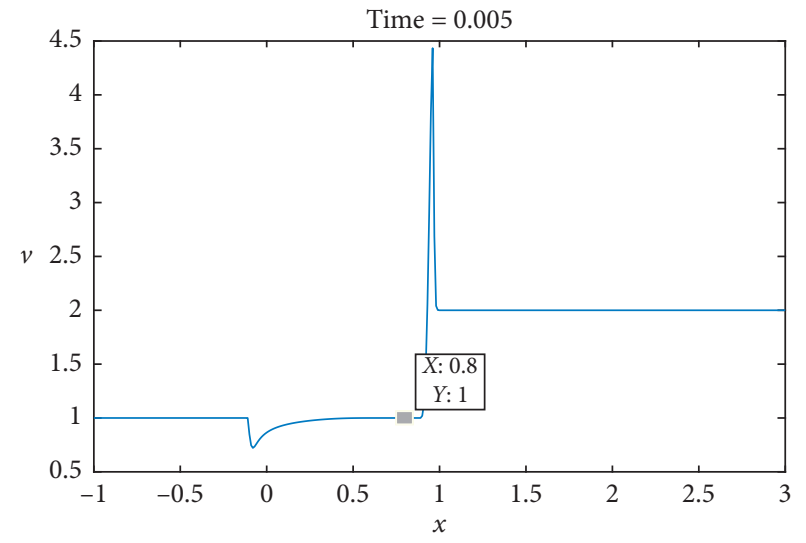

(b)

FiguRE 14: (a) $u$ and (b) $v$ for $v_{m}-u_{m}=0.9$ with $u_{-}=1.5, v_{-}=1, u_{m}=1.9, v_{m}=1, u_{+}=2.3$, and $v_{+}=2$.

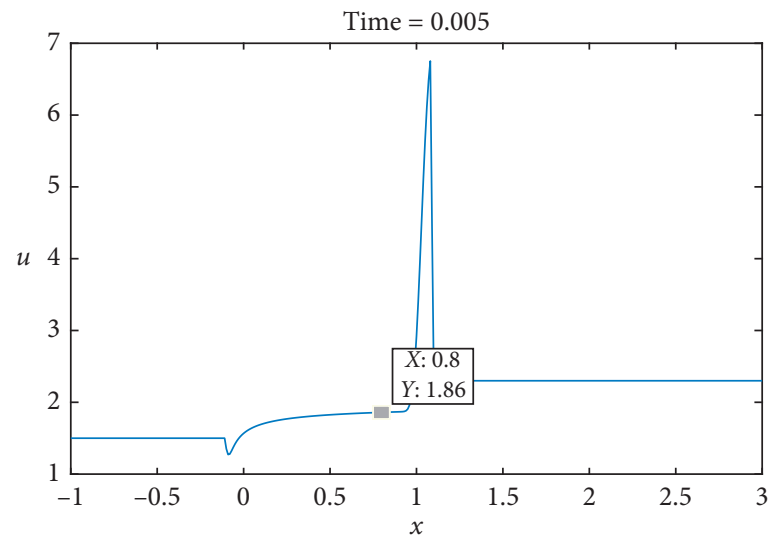

(a)

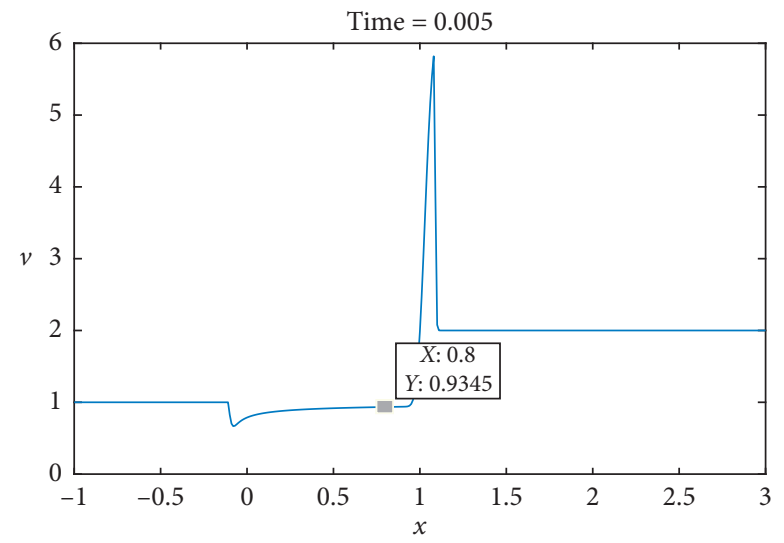

(b)

FIGURE 15: (a) $u$ and (b) $v$ for $v_{m}-u_{m}=0.99$ with $u_{-}=1.5, v_{-}=1, u_{m}=1.99, v_{m}=1, u_{+}=2.3$, and $v_{+}=2$. 


$$
\begin{aligned}
& u_{-}=1.5, \\
& v_{-}=1, \\
& u_{m}=1.9, \\
& v_{m}=1, \\
& u_{+}=2.3, \\
& v_{+}=2, \\
& u_{-}=1.5, \\
& v_{-}=1, \\
& u_{m}=1.99, \\
& v_{m}=1, \\
& u_{+}=2.3, \\
& v_{+}=2 .
\end{aligned}
$$

This implies that the $v_{m}-u_{m}$ from -0.9 (Figure 14) tends to -0.99 (Figure 15). In order to better explain the generation of zero rarefaction wave on the interval $[0,1]$, we subdivide $[-1,3]$ on the space. It can be seen from the following numerical simulations that in a very short period of time, when $v_{-}-u_{-}$approaches -0.99 from -0.9 , the intermediate state $\left(u_{m}, v_{m}\right)=(1.9,1)$ disappears. That is to say, the wave velocity of rarefaction wave $\overrightarrow{R_{1}}$ increases. This is very consistent with our conclusion when $-1<v_{m}-u_{m}<0$ and $v_{m}-u_{m}<\min \left\{v_{-}-u_{-}, v_{+}-u_{+}\right\}$, a zero rarefaction wave is alive in the interval $[0,1]$ when $\left(v_{m}-u_{m}\right) \longrightarrow(-1)^{+}$.

\section{Conclusions}

In this paper, we mainly study the zero waves of the nonsimplified chromatographic equation. From the analysis of Section 3, one can know when $-1<v_{-}-u_{-}<v_{m}-u_{m}<0$, on the one hand, the solution performs as a zero shock wave if $v_{+}-u_{+}<0$ and $\left(v_{-}-u_{-}\right) \longrightarrow(-1)^{+}$. On the other hand, its solution performs as a zero shock wave in the interval $[0,1]$ and a zero delta shock wave in the interval $(1, \infty)$ if $v_{+}-$ $u_{+}>0$ and $\left(v_{-}-u_{-}\right) \longrightarrow(-1)^{+}$. From the analysis of Section 4 , when $-1<v_{-}-u_{-}<0<v_{m}-u_{m}$ and $v_{-}-u_{-}<v_{+}-u_{+}$, the solution performs as a zero delta shock wave when $\left(v_{-}-u_{-}\right) \longrightarrow(-1)^{+}$. From the analysis of Section 5, when $-1<v_{m}-u_{m}<0$ and $v_{m}-u_{m}<\min \left\{v_{-}-u_{-}, v_{+}-u_{+}\right\}$, a zero rarefaction wave is alive in the interval $[0,1]$ when $\left(v_{m}-u_{m}\right) \longrightarrow(-1)^{+}$.

\section{Data Availability}

No data were used to support this study.

\section{Conflicts of Interest}

The authors declare that they have no conflicts of interest.

\section{Acknowledgments}

This work was supported by the National Natural Science Foundation of China (11761068, 11401508, and 11961063) and the Natural Science Foundation of Xinjiang (2017D01C053 and 2019D01C080).

\section{References}

[1] M. Mazzotti, "Occurrence of a delta-shock in non-linear chromatography," Pamm, vol. 7, no. 1, pp. 2040073-2040074, 2007.

[2] M. Mazzotti, "Nonclassical composition fronts in nonlinear chromatography: delta-shock," Industrial \& Engineering Chemistry Research, vol. 48, no. 16, pp. 7733-7752, 2009.

[3] M. Mazzotti, A. Tarafder, J. Cornel, F. Gritti, and G. Guiochon, "Experimental evidence of a delta-shock in nonlinear chromatography," Journal of Chromatography A, vol. 1217, no. 13, pp. 2002-2012, 2010.

[4] G. Wang, "One-dimensional nonlinear chromatography system and delta-shock waves," Zeitschrift für angewandte Mathematik und Physik, vol. 64, no. 5, pp. 1451-1469, 2013.

[5] B. Temple, "Systems of conservation laws with invariant submanifolds," Transactions of the American Mathematical Society, vol. 280, no. 2, p. 781, 1983.

[6] B. Temple, "Global solution of the cauchy problem for a class of $2 \times 2$ nonstrictly hyperbolic conservation laws," Advances in Applied Mathematics, vol. 3, no. 3, pp. 335-375, 1982.

[7] F. Ancona and P. Goatin, "Uniqueness and stability of $L^{\infty}$ solutions for Temple class systems with boundary and properties of the attainable sets," SIAM Journal on Mathematical Analysis, vol. 34, no. 1, pp. 28-63, 2002.

[8] P. Baiti and A. Bressan, "The semigroup generated by a Temple class system with large data," Differential and Integral Equations, vol. 10, pp. 401-418, 1997.

[9] S. Bianchini, "Stability of $L^{\infty}$ solutions for hyperbolic systems with coinciding shocks and rarefactions," SIAM Journal on Mathematical Analysis, vol. 33, no. 4, pp. 959-981, 2001.

[10] A. Bressan and P. Goatin, "Stability of $L^{\infty}$ solutions of Temple class systems," Differential and Integral Equations, vol. 13, pp. 1503-1528, 2000.

[11] Z. Shao, "Riemann problem with delta initial data for the isentropic relativistic Chaplygin Euler equations," Zeitschrift für Angewandte Mathematik und Physik, vol. 69, p. 20, 2018.

[12] Z. Shao, "The Riemann problem for the relativistic full Euler system with generalized Chaplygin proper energy densitypressure relation," Zeitschrift für Angewandte Mathematik und Physik, vol. 67, p. 24, 2016.

[13] M. Sun, "Interactions of elementary waves for the Aw-Rascle model," SIAM Journal on Applied Mathematics, vol. 69, no. 6, pp. 1542-1558, 2009.

[14] L. Ambrosio, G. Crippa, A. Figalli, and L. V. Spinolo, "Some new well-posedness results for continuity and transport equations, and applications to the chromatography system," SIAM Journal on Mathematical Analysis, vol. 41, no. 5, pp. 1890-1920, 2009.

[15] M. Sun, "Delta shock waves for the chromatography equations as self-similar viscosity limits," Quarterly of Applied Mathematics, vol. 69, no. 3, pp. 425-443, 2011.

[16] C. Shen, "The asymptotic behaviors of solutions to the perturbed Riemann problem near the singular curve for the chromatography system," Journal of Nonlinear Mathematical Physics, vol. 22, no. 1, pp. 76-101, 2015.

[17] C. Bourdarias, M. Gisclon, and S. Junca, "Existence of weak entropy solutions for gas chromatography systen with one or two active species and non convex isotherms," Communications in Mathematical Sciences, vol. 5, no. 1, pp. 67-84, 2007. 
[18] H. Cheng and H. Yang, "Delta shock waves in chromatography equations," Journal of Mathematical Analysis and Applications, vol. 380, no. 2, pp. 475-485, 2011.

[19] L. Guo, L. Pan, and G. Yin, “The perturbed Riemann problem and delta contact discontinuity in chromatography equations," Nonlinear Analysis: Theory, Methods \& Applications, vol. 106, pp. 110-123, 2014.

[20] J. Menacho and J. Solà-Morales, "Convergence to steady-state and boundary layer profiles in a linear chromatography system," SIAM Journal on Applied Mathematics, vol. 75, no. 2, pp. 745-761, 2015.

[21] S. M. A. Mohamed and M. Nedeljkov, "Simplified chromatography model and inverse of split delta shocks," Applied Mathematics Letters, vol. 92, pp. 49-53, 2019.

[22] F. Ortner and M. Mazzotti, "Singular shock solutions in nonlinear chromatography," Nonlinear Analysis: Real World Applications, vol. 41, pp. 66-81, 2018.

[23] D. N. Ostrov, "Asymptotic behavior of two interreacting chemicals in a chromatography reactor," SIAM Journal on Mathematical Analysis, vol. 27, no. 6, pp. 1559-1596, 1996.

[24] C. Tsikkou, "Singular shocks in a chromatography model," Journal of Mathematical Analysis and Applications, vol. 439, no. 2, pp. 766-797, 2016.

[25] D. Armbruster, S. Göttlich, and M. Herty, "A scalar conservation law with discontinuous flux for supply chains with finite buffers," SIAM Journal on Applied Mathematics, vol. 71, no. 4, pp. 1070-1087, 2011.

[26] J.-P. Dias and M. Figueira, "On the approximation of the solutions of the Riemann problem for a discontinuous conservation law," Bulletin of the Brazilian Mathematical Society, New Series, vol. 36, no. 1, pp. 115-125, 2005.

[27] S. Göttlich, A. Klar, and P. Schindler, "Discontinuous conservation laws for production networks with finite buffers," SIAM Journal on Applied Mathematics, vol. 73, no. 3, pp. 1117-1138, 2013.

[28] T. Gimse, "Conservation laws with discontinuous flux functions," SIAM Journal on Mathematical Analysis, vol. 24, no. 2, pp. 279-289, 1993.

[29] J. K. Wiens, J. M. Stockie, and J. Williams, "Riemann solver for a kinematic wave traffic model with discontinuous flux," Journal of Computational Physics, vol. 242, pp. 1-23, 2013.

[30] D. Zeidan, P. Bähr, P. Farber, J. Gräbel, and P. Ueberholz, "Numerical investigation of a mixture two-phase flow model in two-dimensional space," Computers \& Fluids, vol. 181, pp. 90-106, 2019.

[31] D. Zeidan, R. Touma, and A. Slaouti, "Implementation of velocity and pressure non-equilibrium in gas-liquid twophase flow computations," International Journal of Fluid Mechanics Research, vol. 41, no. 6, pp. 547-555, 2014.

[32] E. Goncalvès and D. Zeidan, "Numerical simulation of unsteady cavitation in liquid hydrogen flows," International Journal of Engineering Systems Modelling and Simulation, vol. 9, no. 1, pp. 41-51, 2017.

[33] S. Kuila, T. Raja Sekhar, and D. Zeidan, "A Robust and accurate Riemann solver for a compressible two-phase flow model," Applied Mathematics and Computation, vol. 265, pp. 681-695, 2015.

[34] D. Aeidan, E. Ramenski, A. Slaouti, and E. Toro, "Numerical study of wave propagation in compressible two-phase flow," International Journal for Numerical Methods in Fluids, vol. 54, no. 4, pp. 393-418, 2007.

[35] M. Nedeljkov and M. Oberguggenberger, "Interactions of delta shock waves in a strictly hyperbolic system of conservation laws," Journal of Mathematical Analysis and Applications, vol. 344, no. 2, pp. 1143-1157, 2008.

[36] C. Shen and M. Sun, "Interactions of delta shock waves for the transport equations with split delta functions," Journal of Mathematical Analysis and Applications, vol. 351, no. 2, pp. 747-755, 2009.

[37] M. Sun, "Interactions of delta shock waves for the chromatography equations," Applied Mathematics Letters, vol. 26, no. 6, pp. 631-637, 2013. 